\title{
ANALYTIC FAMILIES OF HOLOMORPHIC ITERATED FUNCTION SYSTEMS
}

\author{
MARIO ROY, HIROKI SUMI, AND MARIUSZ URBAŃSKI
}

\begin{abstract}
This paper deals with analytic families of holomorphic iterated function systems. Using real analyticity of the pressure function (which we prove), we establish a classification theorem for analytic families of holomorphic iterated function systems which depend continuously on a parameter when the space of holomorphic iterated function systems is endowed with the $\lambda$-topology. This classification theorem allows us to generalize some geometric results from [17] and gives us a better and clearer understanding of the global structure of the space of conformal IFSs.
\end{abstract}

\section{Introduction}

Iterated function systems (abbreviated to IFSs henceforth) arise in many natural contexts. They are often used to encode and generate fractal images, such as landscapes and skyscapes, in computer games. They each generate, via a recursive procedure, a unique fractal set called attractor or limit set. IFSs also play an important role in the theory of dynamical systems. By dynamical system we mean a continuous map $T$ from a metric space $X$ to itself, where, given $x \in X$, one aims at describing the eventual behavior of the sequence of iterates $\left(T^{n} x\right)_{n=0}^{\infty}$. IFSs are in fact a generalization of the process of looking at the backward trajectories of dynamical systems. A repeller in a dynamical system sometimes coincides with the attractor of an associated IFS. For example, the middle-third Cantor set attracts all the backward orbits of the tent map: $T(x)=3 x$ if $x \leq 1 / 2$ and $T(x)=3(1-x)$ if $x \geq 1 / 2$. This follows from the fact that the inverse branches of $T$ are precisely the generators $\varphi_{1}$ and $\varphi_{2}$ of an IFS. As dynamical systems often model physical processes, the study of IFSs frequently turns out to be instrumental in describing real systems.

The systematic development of modern theory of iterated function systems began with the works of Hutchinson [8], Falconer [5], Barnsley [4], Bandt and Graf [3], and Schief [28], just to name a few. Their pioneering works in the 1980's and 1990's on finite IFSs concerned systems consisting of similarities. In particular, the theory of finite IFSs was used in the study of the complex dynamics of rational functions.

Date: August 16, 2008. Published in Nonlinearity 21 (2008) 2255-2279.

1991 Mathematics Subject Classification. Primary: 37F35; Secondary: 37F45.

Key words and phrases. conformal iterated function system, limit set, Hausdorff dimension, $\lambda$-topology, holomorphic families.

Research of the first author was supported by NSERC (Natural Sciences and Engineering Research Council of Canada). The research of the third author was supported in part by the NSF Grant DMS 0700831. 
In the middle of the 1990's, the need to investigate finite, and even infinite, IFSs that comprise more general conformal maps arose. The foundations of that theory were laid out by Mauldin and Urbański in [12]. Many new applications were also discovered. In 1999, Mauldin and Urbański [13] applied the theory of infinite conformal IFSs to continued fractions with restricted entries. Few years later, Kotus and Urbański [11] applied that theory to obtain a lower estimate for the Hausdorff dimension of the Julia set of elliptic functions, while Urbański and Zdunik [29] obtained similar results in the complex dynamics of the exponential function. They further showed that the framework of conformal IFSs is the right one to study the harmonic measure of various Cantor sets. Applications of conformal IFSs to number theory, more precisely to the theory of continued fractions and Diophantine approximations, were also developed by Urbański in [23], [24], [25], and [26]. Finally, Stratmann and Urbański [21] established extremality in the sense of Kleinbock, Lindenstrauss and Barak Weiss, of conformal measures for convex co-compact Kleinian groups.

A further generalization of the theory was achieved by Mauldin and Urbański [14] in 2003. This generalization relies on graphs. Indeed, every IFS can be thought of as a graph with a single vertex and a countable set of self-loops. To the unique vertex is attached a space $X \subset$ $\mathbb{R}^{d}$. The self-loops represent the generators of the IFS, each generator being a contracting self-map of $X$. The limit set generated by all infinite paths on this graph is also attached to the vertex. Mauldin and Urbański extended the theory of IFSs to graph directed Markov systems (GDMSs). The graphs associated with these systems generally have more than one, though finitely many, vertices. Moreover, they can have a countably infinite set of edges between any two of their vertices. It turns out that a unique fractal set can be associated with each vertex, and the limit set is the disjoint union of those sets. In 2007, Stratmann and Urbański [22] went one step further by laying the foundations to the theory of pseudo-Markov systems. In contradistinction with GDMSs, the underlying graph of these systems can have infinitely many vertices. They applied their work to infinitely generated Schottky groups.

In all those works, the metric structure of the limit set $J$ is usually described by its Hausdorff, packing and/or box-counting dimension(s). The Hausdorff dimension is particularly interesting as it is characterized by the pressure function. The pressure function plays a central role in thermodynamic formalism, which arose from statistical physics. For finite systems which satisfy a certain separation property (the famous open set condition (OSC)), the Hausdorff dimension of the limit set is simply the zero of the pressure function $P(t)$, that is, the unique $t_{0}>0$ such that $P\left(t_{0}\right)=0$. This latter equation is sometimes called Moran-Bowen formula. For infinite systems satisfying the OSC and a bounded distortion property, Mauldin and Urbański [12] showed that a variant of Moran-Bowen formula holds: the Hausdorff dimension of the limit set is the infimum of all $t \geq 0$ for which $P(t) \leq 0$. They also showed that a $t$-conformal measure exists if and only if $P(t)=0$, and there is at most one such $t$.

Recently, interest in families of IFSs and GDMSs has emerged (see [1], [2], [16], [17] and [18], among others). Baribeau and Roy [2] showed that the Hausdorff dimension of the limit set is a continuous, subharmonic function in families of (finite or infinite) IFSs which consist of complex similarities whose coefficients vary holomorphically with a parameter. Using thermodynamic formalism, Roy and Urbański [16] later proved that this result extends to families 
of conformal IFSs. Moreover, they produced diverse families of infinite IFSs in which the Hausdorff dimension of the limit set does not vary real-analytically: there are phase transitions during which the real-analyticity sometimes breaks down. This is in sharp contrast to what happens in the finite case. In a subsequent paper, Roy and Urbański [18] studied the behavior of the Hausdorff dimension function of the limit sets of conformal GDMSs that live in higher dimensional Euclidean spaces $\mathbb{R}^{d}, d \geq 3$. They proved in particular that this function is real-analytic in the subspace of all strongly regular systems that have a finitely irreducible incidence matrix.

Roy and Urbański also considered a slightly different approach in [16]. They fixed an underlying space $X \subset \mathbb{R}^{d}$ and a countable index set $I$, and investigated the space $\operatorname{CIFS}(X, I)$ of all conformal IFSs with alphabet $I$ that live on $X$. When $I$ is finite and $\operatorname{CIFS}(X, I)$ is endowed with the natural metric of pointwise convergence (pointwise meaning that corresponding generators are compared to one another in $\left.C^{1}(X)\right)$, the topological pressure and the Hausdorff dimension functions are continuous functions of the underlying CIFSs. However, they discovered that these functions are generally not continuous when $I$ is infinite (and $\operatorname{CIFS}(X, I)$ is equipped with a metric of pointwise convergence). They thereafter introduced a finer but still natural topology called the $\lambda$-topology, with respect to which the topological pressure and the Hausdorff dimension functions are both continuous (see [16]).

More recently, the authors of the present article have studied more thoroughly the pointwise and $\lambda$-topologies (see [17]). We have shown that the $\lambda$-topology is normal but not metrizable, for it is not first-countable. We studied in detail how various characteristics of an IFS behave with respect to the $\lambda$-topology.

The aim of the current paper is to investigate some families in a subspace of CIFS $(X, I)$ when $d=2$ (or, equivalently, when $X \subset \mathbb{C}^{\text {) }}$ and when $\operatorname{CIFS}(X, I)$ is endowed with the $\lambda$ topology. The abovementioned subspace, denoted by $\operatorname{HIFS}(X, I)$, is the space of all holomorphic CIFSs. We shall study analytic families of HIFSs whose generators depend analytically on a complex parameter.

After some preliminaries on single CIFSs in section 2 and on families of CIFSs in section 3, we study in section 4 the properties of the pressure function seen as a function of two variables, that is, as a function of not only the usual parameter $t$ but also of the underlying IFS $\Phi$. In section 5, we enunciate a classification theorem of analytic families which depend continuously on a parameter when $\operatorname{HIFS}(X, I)$ is endowed with the $\lambda$-topology. Finally, in section 6 , we use our classification theorem to generalize some results that we obtained in [17] and hence to gain a better understanding of $\operatorname{CIFS}(X, I)$ when this latter is equipped with the $\lambda$-topology.

Furthermore, we give some examples of analytic families in $\operatorname{HIFS}(X, I)$ (Example 5.4, Proposition 6.3, Theorem 6.5, Proposition 6.6).

\section{Preliminaries on Iterated Function Systems}

Let us first describe the setting of conformal iterated function systems introduced in [12]. Let $I$ be a countable (finite or infinite) index set (often called alphabet) with at least two elements, and let $\Phi=\left\{\varphi_{i}: X \rightarrow X \mid i \in I\right\}$ be a collection of injective contractions of a 
compact metric space $\left(X, d_{X}\right)$ (sometimes coined seed space) for which there exists a constant $0<s<1$ such that $d_{X}\left(\varphi_{i}(y), \varphi_{i}(x)\right) \leq s d_{X}(y, x)$ for every $x, y \in X$ and for every $i \in I$. Any such collection $\Phi$ is called an iterated function system (abbr. IFS). We define the limit set $J_{\Phi}$ of this system as the image of the coding space $I^{\infty}$ under a coding map $\pi_{\Phi}$, which is defined as follows. Let $I^{n}$ denote the space of words of length $n$ with letters in $I$, let $I^{*}:=\bigcup_{n \in \mathbb{N}} I^{n}$ be the space of finite words, and $I^{\infty}$ the space of one-sided infinite words with letters in $I$. Given $\omega, \tau \in I^{\infty}$, we define $\omega \wedge \tau \in I^{*} \cup I^{\infty}$ to be the longest initial block common to both $\omega$ and $\tau$. For every $\omega \in I^{*} \cup I^{\infty}$, we write $|\omega|$ for the length of $\omega$, that is, the unique $n \in \mathbb{N} \cup\{\infty\}$ such that $\omega \in I^{n}$. For $\omega \in I^{n}$ with $n \in \mathbb{N}$, we set $\varphi_{\omega}:=\varphi_{\omega_{1}} \circ \varphi_{\omega_{2}} \circ \cdots \circ \varphi_{\omega_{n}}$. If $\omega \in I^{*} \cup I^{\infty}$ and $n \in \mathbb{N}$ does not exceed the length of $\omega$, we denote by $\left.\omega\right|_{n}$ the word $\omega_{1} \omega_{2} \ldots \omega_{n}$. Since, given $\omega \in I^{\infty}$, the diameters of the compact sets $\varphi_{\left.\omega\right|_{n}}(X), n \in \mathbb{N}$, converge to zero and since these sets form a decreasing family, their intersection

$$
\bigcap_{n=1}^{\infty} \varphi_{\left.\omega\right|_{n}}(X)
$$

is a singleton, and we denote its element by $\pi_{\Phi}(\omega)$. This defines the coding map $\pi_{\Phi}: I^{\infty} \rightarrow X$. Clearly, $\pi_{\Phi}$ is a continuous function when $I^{\infty}$ is equipped with the topology generated by the cylinders $[i]_{n}=\left\{\omega \in I^{\infty}: \omega_{n}=i\right\}, i \in I, n \in \mathbb{N}$. The main object of our interest is the limit set

$$
J_{\Phi}=\pi_{\Phi}\left(I^{\infty}\right)=\bigcup_{\omega \in I^{\infty}} \bigcap_{n=1}^{\infty} \varphi_{\left.\omega\right|_{n}}(X) .
$$

Observe that $J_{\Phi}$ satisfies the natural invariance property, $J_{\Phi}=\bigcup_{i \in I} \varphi_{i}\left(J_{\Phi}\right)$. Note also that if $I$ is finite, then $J_{\Phi}$ is compact, which is usually not the case when $I$ is infinite.

An IFS $\Phi=\left\{\varphi_{i}\right\}_{i \in I}$ is said to satisfy the Open Set Condition (OSC) if there exists a nonempty open set $U \subset X$ such that $\varphi_{i}(U) \subset U$ for every $i \in I$ and $\varphi_{i}(U) \cap \varphi_{j}(U)=\emptyset$ for every pair $i, j \in I, i \neq j$.

An IFS $\Phi$ is called conformal (and thereafter a CIFS) if $X$ is connected, $X=\overline{\operatorname{Int}_{\mathbb{R}^{d}}(X)}$ for some $d \in \mathbb{N}$, and if the following conditions are satisfied:

(i) $\Phi$ satisfies the OSC with $U=\operatorname{Int}_{\mathbb{R}^{d}}(X)$;

(ii) There exists an open connected set $V$, with $X \subset V \subset \mathbb{R}^{d}$, such that all the generators $\varphi_{i}, i \in I$, extend to $C^{1}$ conformal diffeomorphisms of $V$ into $V$;

(iii) There exist $\gamma, l>0$ such that for every $x \in X$ there is an open cone $\operatorname{Con}(x, \gamma, l) \subset$ $\operatorname{Int}(X)$ with vertex $x$, central angle of Lebesgue measure $\gamma$, and altitude $l$;

(iv) There are two constants $L \geq 1$ and $\alpha>0$ such that

$$
|| \varphi_{i}^{\prime}(y)|-| \varphi_{i}^{\prime}(x)|| \leq L\left\|\left(\varphi_{i}^{\prime}\right)^{-1}\right\|_{V}^{-1}\|y-x\|^{\alpha}
$$

for all $x, y \in V$ and all $i \in I$, where $\|\cdot\|_{V}$ is the supremum norm taken over $V$. 
Remark 2.1. It has been proved in Proposition 4.2.1 of [14] that if $d \geq 2$, then condition (iv) is satisfied with $\alpha=1$. This condition is also fulfilled if $d=1$, the alphabet $I$ is finite and all the maps $\varphi_{i}$ are of class $C^{1+\varepsilon}$.

The following useful fact has been also proved in Lemma 4.2.2 of [14].

Lemma 2.2. For all $\omega \in I^{*}$ and all $x, y \in V$ we have that

$$
|\log | \varphi_{\omega}^{\prime}(y)|-\log | \varphi_{\omega}^{\prime}(x)|| \leq L(1-s)^{-1}\|y-x\|^{\alpha} .
$$

As an immediate consequence of this lemma we get the following.

(iv') Bounded Distortion Property (BDP): There exists a constant $K \geq 1$ such that

$$
\left|\varphi_{\omega}^{\prime}(y)\right| \leq K\left|\varphi_{\omega}^{\prime}(x)\right|
$$

for every $x, y \in V$ and every $\omega \in I^{*}$, where $\left|\varphi_{\omega}^{\prime}(x)\right|$ denotes the norm of the derivative.

As demonstrated in [12], infinite CIFSs, unlike finite ones, split naturally into two main classes: irregular and regular systems. This dichotomy can be expressed in terms of the absence or existence of a zero for the topological pressure function. Recall that the topological pressure $\mathrm{P}_{\Phi}(t), t \geq 0$, is defined as follows. For every $n \in \mathbb{N}$, set

$$
\mathrm{P}_{\Phi}^{(n)}(t)=\sum_{\omega \in I^{n}}\left\|\varphi_{\omega}^{\prime}\right\|^{t}
$$

where $\|\cdot\|:=\|\cdot\|_{X}$ is the supremum norm over $X$. Then

$$
\mathrm{P}_{\Phi}(t)=\lim _{n \rightarrow \infty} \frac{1}{n} \log \mathrm{P}_{\Phi}^{(n)}(t)=\inf _{n \in \mathbb{N}} \frac{1}{n} \log \mathrm{P}_{\Phi}^{(n)}(t) .
$$

Recall also that the shift map $\sigma: I^{*} \cup I^{\infty} \rightarrow I^{*} \cup I^{\infty}$ is defined for each $\omega \in I^{*} \cup I^{\infty}$ as

$$
\sigma\left(\left\{\omega_{n}\right\}_{n=1}^{|\omega|}\right)=\left\{\omega_{n+1}\right\}_{n=1}^{|\omega|-1} .
$$

Now, if the function $\zeta_{\Phi}: I^{\infty} \rightarrow \mathbb{R}$ is given by the formula

$$
\zeta_{\Phi}(\omega)=\log \left|\varphi_{\omega_{1}}^{\prime}(\pi(\sigma(\omega)))\right|
$$

then $\mathrm{P}_{\Phi}(t)=\mathrm{P}\left(t \zeta_{\Phi}\right)$, where $\mathrm{P}\left(t \zeta_{\Phi}\right)$ is the classical topological pressure of the function $t \zeta_{\Phi}$ when $I$ is finite (so the space $I^{\infty}$ is compact), and is understood in the sense of [7] and [14] when $I$ is infinite. The finiteness parameter $\theta_{\Phi}$ of a system is defined as $\inf \left\{t \geq 0: \mathrm{P}_{\Phi}^{(1)}(t)<\right.$ $\infty\}=\inf \left\{t \geq 0: \mathrm{P}_{\Phi}(t)<\infty\right\}$. Let also

$$
\operatorname{Fin}(\Phi)=\left\{t \geq 0: P_{\Phi}(t)<\infty\right\}=\left\{t \geq 0: P_{\Phi}^{(1)}(t)<\infty\right\} .
$$

In [12], it was shown that the topological pressure function $\mathrm{P}_{\Phi}$ is non-increasing on $[0, \infty)$, (strictly) decreasing, continuous and convex on $\left[\theta_{\Phi}, \infty\right)$, and $\mathrm{P}_{\Phi}(d) \leq 0$. Of course, $\mathrm{P}_{\Phi}(0)=\infty$ 
if and only if $I$ is infinite. The following characterization of the Hausdorff dimension $h_{\Phi}$ of the limit set $J_{\Phi}$ was stated as Theorem 3.15 in [12]. For every $F \subset I$, we write $\left.\Phi\right|_{F}$ for the subsystem $\left\{\varphi_{i}\right\}_{i \in F}$ of $\Phi$.

\section{Theorem 2.3.}

$$
h_{\Phi}=\sup \left\{h_{\left.\Phi\right|_{F}}: F \subset I \text { is finite }\right\}=\inf \left\{t \geq 0: \mathrm{P}_{\Phi}(t) \leq 0\right\} .
$$

In particular, if $\mathrm{P}_{\Phi}(t)=0$, then $t=h_{\Phi}$.

Subsequently, a system $\Phi$ was called regular provided there is some $t \geq 0$ such that $\mathrm{P}_{\Phi}(t)=0$. It follows from the strict decrease of $P_{\Phi}$ on $\left[\theta_{\Phi}, \infty\right)$ that such a $t$ is unique, if it exists. If no such $t$ exists, the system is called irregular.

Regular systems can also be naturally divided into subclasses. Following [12] still, a system $\Phi$ is said to be strongly regular if $0<\mathrm{P}_{\Phi}(t)<\infty$ for some $t \geq 0$. As an immediate application of Theorem 2.3, a system $\Phi$ is strongly regular if and only if $h_{\Phi}>\theta_{\Phi}$. A system $\Phi$ is called cofinitely regular provided that every nonempty cofinite subsystem $\Phi^{\prime}=\left\{\varphi_{i}\right\}_{i \in I^{\prime}}$ (with $I^{\prime}$ any cofinite subset of $I$ ) is regular. A finite system is clearly cofinitely regular, and it was shown in [12] that an infinite system is cofinitely regular exactly when the pressure is infinite at the finiteness parameter, that is, $\mathrm{P}_{\Phi}\left(\theta_{\Phi}\right)=\infty$. Note that every cofinitely regular system is strongly regular, and every strongly regular system is regular. Finally, recall from [16] that critically regular systems are those regular systems for which $P_{\Phi}\left(\theta_{\Phi}\right)=0$.

\section{Preliminaries on Families of CiFSs}

When dealing with families of CIFSs, we denote the set of all conformal iterated function systems with phase space $X$ and alphabet $I$ by $\operatorname{CIFS}(X, I)$. Obviously, $\operatorname{CIFS}(X, I)$ can be endowed with different topologies.

When $I$ is finite, $\operatorname{CIFS}(X, I)$ is naturally endowed with the metric of pointwise convergence. This metric asserts that the distance between CIFSs $\Phi=\left\{\varphi_{i}\right\}_{i \in I}$ and $\Psi=\left\{\psi_{i}\right\}_{i \in I}$ is

$$
\rho(\Phi, \Psi)=\sum_{i \in I}\left(\left\|\varphi_{i}-\psi_{i}\right\|+\left\|\varphi_{i}^{\prime}-\psi_{i}^{\prime}\right\|\right)
$$

It was proved in [16] that, given $t \geq 0$, the pressure function $P(t): \operatorname{CIFS}(X, I) \rightarrow \mathbb{R}$, $\Phi \mapsto P_{\Phi}(t)$ and the Hausdorff dimension function $h: \operatorname{CIFS}(X, I) \rightarrow[0, d], \Phi \mapsto h_{\Phi}$ are continuous (see Lemma 4.2 and Theorem 4.3 in [16], respectively).

When $I$ is infinite, the situation is more intricate. First, recall that we may assume that $I=\mathbb{N}$ without loss of generality. Henceforth, we accordingly abbreviate $\operatorname{CIFS}(X, I N)$ to $\operatorname{CIFS}(X)$. The set $\operatorname{CIFS}(X)$ can easily be equipped with a metric of pointwise convergence. In [16], such a metric was introduced by defining the distance between two CIFSs $\Phi$ and $\Psi$ as

$$
\rho_{\infty}(\Phi, \Psi)=\sum_{i=1}^{\infty} 2^{-i} \min \left\{1,\left\|\varphi_{i}-\psi_{i}\right\|+\left\|\varphi_{i}^{\prime}-\psi_{i}^{\prime}\right\|\right\} .
$$


In these definitions, $\|\cdot\|:=\|\cdot\|_{X}$ stands for the supremum norm over $X$.

Roy and Urbański observed that the pressure and Hausdorff dimension functions are generally not continuous when $\operatorname{CIFS}(X)$ is endowed with the metric $\rho_{\infty}$ (see the example following Lemma 5.3 in [16]). This triggered the introduction of a topology called the $\lambda$-topology (see section 5 in [16]). In that topology, a sequence $\left\{\Phi^{n}\right\}$ converges to $\Phi$ provided that $\left\{\Phi^{n}\right\}$ converges to $\Phi$ in the pointwise topology and that there exist constants $C>0$ and $N \in \mathbb{I}$ such that

$$
\left|\log \left\|\varphi_{i}^{\prime}\right\|-\log \left\|\left(\varphi_{i}^{n}\right)^{\prime}\right\|\right| \leq C
$$

for all $i \in \mathbb{N}$ and all $n \geq N$. A set $F \subset \operatorname{CIFS}(X)$ is declared to be closed if the $\lambda$-limit of every $\lambda$-converging sequence of CIFSs in $F$ belongs to $F$. Several topological properties of $\operatorname{CIFS}(X)$ were given in [16] and [17]. Among others, let us mention that this topology is not metrizable, for it does not even satisfy the first axiom of countability (see Proposition 5.7 in [17]). Nonetheless, this topology proves to be useful, for it is easy to determine whether a sequence converges or not in that topology and, according to Roy and Urbański, the Hausdorff dimension function is then continuous everywhere on $\operatorname{CIFS}(X)$ (see Theorem 5.10 in [16]). In fact, the combination of Theorem 5.7 in [16] with Lemma 5.22 in [17] shows that the pressure function is continuous wherever it possibly can be.

Whichever topology we choose to endow $\operatorname{CIFS}(X)$ with, there are some subspaces of particular interest. Following the notation in [17], let $\operatorname{SIFS}(X)$ represent the subset of $\operatorname{CIFS}(X)$ comprising all similarity iterated function systems, that is, iterated function systems whose generators are similarities. Let also $\operatorname{IR}(X) \subset \operatorname{CIFS}(X)$ be the subset of irregular systems, while $\mathrm{R}(X) \subset \operatorname{CIFS}(X)$ will represent the subset of regular systems. Denote further by $\mathrm{CR}(X) \subset \mathrm{R}(X)$ the subset of critically regular systems, by $\mathrm{SR}(X) \subset \mathrm{R}(X)$ the subset of strongly regular systems, and by $\mathrm{CFR}(X) \subset \mathrm{SR}(X)$ the subset of cofinitely regular systems. Finally, we will denote by $\operatorname{FSR}(X)$ the set $\mathrm{SR}(X) \backslash \mathrm{CFR}(X)$.

In this paper, we will concentrate on the case $d=2$ or, equivalently, $X \subset \mathbb{C}$. In this case, a natural subset of $\operatorname{CIFS}(X, I)$ is:

Definition 3.1. The set $\operatorname{HIFS}(X, I)$ consists of those systems $\Phi=\left\{\varphi_{i}\right\}_{i \in I} \in \operatorname{CIFS}(X, I)$ which admit an open connected neighborhood $V$ of $X \subset \mathbb{C}$ such that for each $i \in I$, the map $\varphi_{i}$ extends to a holomorphic diffeomorphism of $V$ into $V$.

More specifically, we will be interested in analytic families of HIFSs.

Definition 3.2. A family $\left\{\Phi^{\gamma}\right\}_{\gamma \in \Gamma}=\left\{\left\{\varphi_{i}^{\gamma}\right\}_{i \in I}\right\}_{\gamma \in \Gamma}$ in $\operatorname{HIFS}(X, I)$ is called analytic if

- $\Gamma$ is a connected, finite-dimensional complex manifold; and

- for each $\gamma_{0} \in \Gamma$, there exists a neighborhood $\Gamma_{0}$ of $\gamma_{0}$ and a bounded connected open neighborhood $V_{0}$ of $X \subset \mathbb{C}$ such that for each $i \in I$ and each $\gamma \in \Gamma$, $\varphi_{i}^{\gamma}$ extends to a holomorphic diffeomorphism of $V_{0}$ into $V_{0}$, and for each $i \in I$, the map

$$
(\gamma, x) \mapsto \varphi_{i}^{\gamma}(x), \quad(\gamma, x) \in \Gamma_{0} \times V_{0},
$$

is holomorphic. 


\section{The Pressure as a Function of Two Variables}

In this section, we make some observations about the pressure function seen as a function of two variables, that is, as a function of not only the parameter $t$ but also of the underlying CIFS $\Phi$. These observations mostly follow from earlier results presented in [16] and [17].

When the alphabet $I$ is finite, the pressure behaves well.

Lemma 4.1. If $I$ is a finite alphabet, then the pressure function $(\Phi, t) \mapsto P(\Phi, t)=P\left(t \zeta_{\Phi}\right)$, $\Phi \in \operatorname{CIFS}(X, I), t \geq 0$, is continuous.

Proof. In the proof of Lemma 4.2 in [16], it was shown that the function $\Phi \mapsto \zeta_{\Phi} \in C\left(I^{\infty}\right)$ is continuous. Since the pressure function $P: C\left(I^{\infty}\right) \rightarrow \mathbb{R}$ is Lipschitz continuous with Lipschitz constant 1 , we deduce that

$$
\begin{aligned}
\left|P(\Phi, t)-P\left(\Phi_{0}, t_{0}\right)\right| & =\left|P\left(t \zeta_{\Phi}\right)-P\left(t_{0} \zeta_{\Phi_{0}}\right)\right| \\
& \leq\left\|t \zeta_{\Phi}-t_{0} \zeta_{\Phi_{0}}\right\| \\
& \leq\left\|t \zeta_{\Phi}-t \zeta_{\Phi_{0}}\right\|+\left\|t \zeta_{\Phi_{0}}-t_{0} \zeta_{\Phi_{0}}\right\| \\
& =|t| \cdot\left\|\zeta_{\Phi}-\zeta_{\Phi_{0}}\right\|+\left|t-t_{0}\right| \cdot\left\|\zeta_{\Phi_{0}}\right\|,
\end{aligned}
$$

for every $\Phi, \Phi_{0} \in \operatorname{CIFS}(X, I)$ and $t, t_{0} \geq 0$. Thus, the pressure function $(\Phi, t) \mapsto P(\Phi, t)$ is continuous.

For an infinite alphabet, we obtain immediately the following. Recall that for any $\Phi \in$ $\operatorname{CIFS}(X)$,

$$
\operatorname{Fin}(\Phi)=\{t \geq 0: P(\Phi, t)<\infty\}
$$

Lemma 4.2. The pressure function $(\Phi, t) \mapsto P(\Phi, t), \Phi \in \operatorname{CIFS}(X), t \geq 0$, is lower semicontinuous when $\operatorname{CIFS}(X)$ is endowed with the metric $\rho_{\infty}$ of pointwise convergence. Moreover, it is continuous on the set $\cup_{\Phi \in \operatorname{CIFS}(X)}\{\Phi\} \times F i n(\Phi)^{c}$, and discontinuous on $\cup_{\Phi \in \operatorname{CIFS}(X)}\{\Phi\} \times$ $(F i n(\Phi) \cap[0, d))$. In fact, in this latter case, given any $\Phi \in \operatorname{CIFS}(X)$ and $t \in F i n(\Phi) \cap[0, d)$, the function $\Psi \mapsto P(\Psi, t)$ is discontinuous at $\Phi$.

Proof. Using Theorem 2.1.5 in [14] as well as Lemma 4.1, for any finite subset $F$ of $\mathbb{I N}$ we have that

$$
\liminf _{(\Phi, t) \rightarrow\left(\Phi_{0}, t_{0}\right)} P(\Phi, t) \geq \liminf _{(\Phi, t) \rightarrow\left(\Phi_{0}, t_{0}\right)} P_{F}(\Phi, t)=\lim _{(\Phi, t) \rightarrow\left(\Phi_{0}, t_{0}\right)} P_{F}(\Phi, t)=P_{F}\left(\Phi_{0}, t_{0}\right) .
$$

Since this is true for every finite subset $F$ of $\mathbb{I N}$, we deduce from Theorem 2.1.5 in [14] that

$$
\liminf _{(\Phi, t) \rightarrow\left(\Phi_{0}, t_{0}\right)} P(\Phi, t) \geq \sup _{F \subset \mathbb{N}, F \text { finite }} P_{F}\left(\Phi_{0}, t_{0}\right)=P\left(\Phi_{0}, t_{0}\right) .
$$

Observe also that $P(\Phi, t)=\infty$ for any $t \notin F i n(\Phi)$ and every $\Phi \in \operatorname{CIFS}(X)$. Thus, the pressure function is upper semi-continuous at any point in the set $\cup_{\Phi \in \operatorname{CIFS}(X)}\{\Phi\} \times F i n(\Phi)^{c}$.

Finally, given $\Phi \in \operatorname{CIFS}(X)$ and $t \in \operatorname{Fin}(\Phi) \cap[0, d)$, the function $\Psi \mapsto P(\Psi, t)$ is discontinuous at $\Phi$ according to the last paragraph in the proof of Lemma 4.4 in [17]. 
When $\operatorname{CIFS}(X)$ is endowed with the $\lambda$-topology, we have the following result.

Lemma 4.3. The pressure function $(\Phi, t) \mapsto P(\Phi, t)$ is continuous on the set $\cup_{\Phi \in \mathrm{CIFS}(X)}\{\Phi\} \times$ Fin $(\Phi)^{c}$ and sequentially continuous on $\cup_{\Phi \in \operatorname{CIFS}(X)}\{\Phi\} \times F i n(\Phi)$ when $\operatorname{CIFS}(X)$ is endowed with the $\lambda$-topology.

Proof. The continuity of the pressure on the set $\cup_{\Phi \in \operatorname{CIFS}(X)}\{\Phi\} \times \operatorname{Fin}(\Phi)^{c}$ follows from Lemma 4.2. The sequential continuity on $\cup_{\Phi \in \operatorname{CIFS}(X)}\{\Phi\} \times F i n(\Phi)$ follows from Lemma 5.22 in [17] and the fact that if $\left\{\Phi^{n}\right\}$ converges to $\Phi$ in the $\lambda$-topology, then $\operatorname{Fin}\left(\Phi^{n}\right)=\operatorname{Fin}(\Phi)$ for all $n$ large enough, as observed before Theorem 5.20 in [17].

We now investigate the properties of the pressure function $(\gamma, t) \mapsto P(\gamma, t)=P\left(\Phi^{\gamma}, t\right)$ within analytic families in $\operatorname{HIFS}(X, I)$.

We first prove that every analytic family in $\operatorname{HIFS}(X, I)$ constitutes a continuous family in $\operatorname{CIFS}(X, I)$ when this latter is equipped with the pointwise topology.

Theorem 4.4. An analytic family $\left\{\Phi^{\gamma}\right\}_{\gamma \in \Gamma}$ regarded as the function

$$
\begin{array}{cccc}
\Phi: \Gamma & \rightarrow & \operatorname{CIFS}(X, I) \\
\gamma & \mapsto & \Phi^{\gamma}
\end{array}
$$

is continuous when $\operatorname{CIFS}(X, I)$ is endowed with the metric of pointwise convergence ( $\rho$ or $\rho_{\infty}$ depending on the case).

Proof. The proof in the infinite case reduces to the finite case. Indeed, if $\Phi$ were discontinuous at some $\gamma_{0} \in \Gamma$, there would exist $\varepsilon>0$ and a sequence $\left\{\gamma_{n}\right\}$ such that $\gamma_{n} \rightarrow \gamma_{0}$ but $\rho_{\infty}\left(\Phi^{\gamma_{n}}, \Phi^{\gamma_{0}}\right) \geq \varepsilon$ for every $n \in \mathbb{N}$. Choose $J \in \mathbb{N}$ such that $\sum_{j>J} 2^{-j}<\varepsilon / 2$. Then

$$
\sum_{j \leq J} 2^{-j} \min \left\{1,\left\|\varphi_{j}^{\gamma_{n}}-\varphi_{j}^{\gamma_{0}}\right\|+\left\|\left(\varphi_{j}^{\gamma_{n}}\right)^{\prime}-\left(\varphi_{j}^{\gamma_{0}}\right)^{\prime}\right\|\right\}>\varepsilon / 2
$$

since, otherwise, we would have $\rho_{\infty}\left(\Phi^{\gamma_{n}}, \Phi^{\gamma_{0}}\right)<\varepsilon$. Replacing the sequence $\left\{\gamma_{n}\right\}$ by one of its subsequences if necessary, it follows from this that there is an $i \leq J$ such that

$$
\left\|\varphi_{i}^{\gamma_{n}}-\varphi_{i}^{\gamma_{0}}\right\|+\left\|\left(\varphi_{i}^{\gamma_{n}}\right)^{\prime}-\left(\varphi_{i}^{\gamma_{0}}\right)^{\prime}\right\|>\frac{\varepsilon}{2 J}
$$

for every $n \in \mathbb{N}$. Consequently, each subsequence of $\left\{\varphi_{i}^{\gamma_{n}}\right\}_{n \in \mathbb{N}}$ either does not converge uniformly on $X$ to $\varphi_{i}^{\gamma_{0}}$ or the corresponding subsequence of derivatives does not converge uniformly on $X$ to $\left(\varphi_{i}^{\gamma_{0}}\right)^{\prime}$.

However, given the neighborhood $V_{0}$ from the definition of an analytic family, the generators $\left\{\varphi_{j}^{\gamma}\right\}_{j \in \mathbb{N}, \gamma \in \Gamma}$ form a family of holomorphic maps uniformly bounded on $V_{0}$, say by a constant $M$, due to the forward invariance of $V_{0}$. By Cauchy's Integral Formula, their derivatives are thereby uniformly bounded on any compact subset $K$ of $V_{0}$ by a constant that depends on $M, K$ and $V_{0}$ only (but neither on $j$ nor on $\gamma$ ). Therefore the generators constitute an equicontinuous family on $V_{0}$ and thereby form a normal family by Arzelà-Ascoli's Theorem. Consequently, the sequence $\left\{\varphi_{i}^{\gamma_{n}}\right\}_{n \in \mathbb{N}}$ admits a subsequence that converges uniformly on an 
open neighborhood $U$ of $X$ which is relatively compact in $V_{0}$. By Hurwitz's Theorem, so will the associated derivatives subsequence. Moreover, in any analytic family, the function $\gamma \mapsto \varphi_{j}^{\gamma}(x), \gamma \in \Gamma$, is holomorphic for each $x \in V_{0}$ and $j \in \mathbb{N}$. Thus, the sequence $\left\{\varphi_{i}^{\gamma_{n}}\right\}_{n \in \mathbb{N}}$ converges pointwise to $\varphi_{i}^{\gamma_{0}}$ on $V_{0}$. Hence $\left\{\varphi_{i}^{\gamma_{n}}\right\}_{n \in \mathbb{N}}$ has a subsequence that converges uniformly on $U$ to $\varphi_{i}^{\gamma_{0}}$. The derivatives subsequence $\left\{\left(\varphi_{i}^{\gamma_{n}}\right)^{\prime}\right\}_{n \in \mathbb{N}}$ will then converge uniformly to $\left(\varphi_{i}^{\gamma_{0}}\right)^{\prime}$ on $U$, and in particular on $X$. This is a contradiction.

In light of Theorem 4.4, it is relevant to ask whether an analytic family is continuous when $\operatorname{CIFS}(X)$ is endowed with the $\lambda$-topology.

Remark 4.5. An analytic family $\left\{\Phi^{\gamma}\right\}_{\gamma \in \Gamma}$ regarded as the function

$$
\begin{aligned}
\Phi: \Gamma & \rightarrow \operatorname{CIFS}(X) \\
\gamma & \mapsto \Phi^{\gamma}
\end{aligned}
$$

is generally not continuous when $\operatorname{CIFS}(X)$ is endowed with the $\lambda$-topology.

Indeed, if this were the case then the finiteness parameter function $\gamma \mapsto \theta_{\Phi^{\gamma}}$ would be locally constant according to Lemma 5.4 in [16]. Since $\Gamma$ is connected, that function would in fact be constant. However, Example 6 in section 8 of [16] presents an analytic family whose finiteness parameter varies.

An immediate consequence of Theorem 4.4 is the following.

Corollary 4.6. Within an analytic family $\left\{\Phi^{\gamma}\right\}_{\gamma \in \Gamma}$, the pressure function $(\gamma, t) \mapsto P(\gamma, t):=$ $P\left(\Phi^{\gamma}, t\right)$, is lower semi-continuous when $\operatorname{CIFS}(X)$ is endowed with the pointwise topology. Moreover, it is continuous on the set $\cup_{\gamma \in \Gamma}\{\gamma\} \times F i n\left(\Phi^{\gamma}\right)^{c}$.

Proof. It follows immediately from Theorem 4.4 that the map $(\gamma, t) \mapsto\left(\Phi^{\gamma}, t\right)$ is continuous, while Lemma 4.2 asserts that the map $(\Phi, t) \mapsto P(\Phi, t)$ is lower semi-continuous. The composition of these two functions, the pressure function $(\gamma, t) \mapsto P(\gamma, t):=P\left(\Phi^{\gamma}, t\right)$, is thus lower semi-continuous. Observe also that $P(\gamma, t)=\infty$ for any $t \notin F i n\left(\Phi^{\gamma}\right)$ and every $\gamma \in \Gamma$. Thus, the pressure function is upper semi-continuous at any point of the set $\cup_{\gamma \in \Gamma}\{\gamma\} \times F i n\left(\Phi^{\gamma}\right)^{c}$.

Remark 4.7. Note that $(\gamma, t) \mapsto P(\gamma, t)$ may be discontinuous at some points in $\cup_{\gamma \in \Gamma}\{\gamma\} \times$ Fin $\left(\Phi^{\gamma}\right)$. For instance, Examples 1, 2 and 3 in section 8 of [16] show that the function $\gamma \mapsto P(\gamma, t)$ may be discontinuous at $t=\theta_{\Phi^{\gamma}}$ if $\Phi^{\gamma} \notin \mathrm{CFR}(X)$. In all three examples, this function is discontinuous on the set $\{(\gamma, t) \in \mathbb{C} \times \mathbb{R}:|\gamma|=1, t=1\}$.

We further have the following result when $I$ is finite.

Theorem 4.8. Let $I$ be a finite alphabet. If $\left\{\Phi^{\gamma}\right\}_{\gamma \in \Gamma}$ is an analytic family in $\operatorname{HIFS}(X, I)$, then for every $t \geq 0$ the pressure function $\gamma \mapsto P(\gamma, t)$ is a continuous, plurisubharmonic function. 
Proof. Fix $t \geq 0$. Since $I$ is finite, each $\Phi^{\gamma}$ is regular. According to the proof of Theorem 6.3 in [16], fixing any $\gamma_{0} \in \Gamma$, we have that

$$
P(\gamma, t)=\sup _{\mu \in M\left(\gamma_{0}\right)}\left\{h_{\mu}(\sigma)+t \int_{I^{\infty}} \zeta_{\Phi^{\gamma}}(\omega) d \mu(\omega)\right\}
$$

and the function $\gamma \mapsto \int_{I^{\infty}} \zeta_{\Phi^{\gamma}}(\omega) d \mu(\omega)$ is finite, positive and pluriharmonic for every $\mu \in$ $M\left(\gamma_{0}\right)$. Thus, $P(\gamma, t)$ is the supremum of a family of pluriharmonic functions that are uniformly bounded from above by $\log |I|$. Therefore $P(\gamma, t)$ is a plurisubharmonic function uniformly bounded from above by $\log |I|$.

In the infinite case, analytic families that are continuous with respect to the $\lambda$-topology on $\operatorname{HIFS}(X)$ enjoy the following property.

Theorem 4.9. Let $t \geq 0$ and $\left\{\Phi^{\gamma}\right\}_{\gamma \in \Gamma}$ be an analytic family in $\operatorname{HIFS}(X)$ such that $\gamma \mapsto \Phi^{\gamma} \in$ $\operatorname{HIFS}(X)$ is continuous with respect to the $\lambda$-topology. Suppose that there exists $\gamma_{0} \in \Gamma$ such that $P\left(\gamma_{0}, t\right)<\infty$. Then the function $\gamma \mapsto P(\gamma, t), \gamma \in \Gamma$, is continuous and plurisubharmonic. In particular, this function satisfies the Maximum Principle.

Proof. Using Theorem 5.7 in [16], $\gamma \mapsto P(\gamma, t)$ is continuous. Moreover, using Theorem 2.1.5 in [14], the Variational Principle and Fubini's Theorem, it follows that the function $\gamma \mapsto$ $P(\gamma, t)$ is a supremum of a family of pluriharmonic functions. Hence it is plurisubharmonic.

4.1. Real Analyticity of Pressure. Since all the forthcoming results rely upon the theorem below and since its proof is somewhat lengthy we single it out in this subsection. Moreover, in order to allege notation we will write $\pi_{\gamma}$ for $\pi_{\Phi^{\gamma}}$ and $P(\gamma, t)$ for $P\left(\Phi^{\gamma}, t\right)$.

Theorem 4.10. Let $\left\{\Phi^{\gamma}\right\}_{\gamma \in \Gamma}$ be an analytic family in $\operatorname{HIFS}(X)$ with the following property.

- For every $\gamma_{0} \in \Gamma$ there exist a neighborhood $\Gamma_{0}$ of $\gamma_{0}$ and $B \geq 0$ such that

$$
\left|\frac{\left(\varphi_{\omega_{1}}^{\gamma}\right)^{\prime}\left(\pi_{\gamma}(\sigma(\omega))\right)}{\left(\varphi_{\omega_{1}}^{\gamma_{0}}\right)^{\prime}\left(\pi_{\gamma_{0}}(\sigma(\omega))\right)}\right| \leq B
$$

for every $\omega \in I^{\infty}$ and all $\gamma \in \Gamma_{0}$.

If $t \geq 0$ and $P\left(\gamma_{1}, t\right)<\infty$ for some $\gamma_{1} \in \Gamma$, then $P(\gamma, t)<\infty$ for all $\gamma \in \Gamma$ and the function $\gamma \mapsto P(\gamma, t), \gamma \in \Gamma$, is real-analytic.

In the case $\operatorname{dim}(\Gamma)=1$ the proof of this theorem is contained in the proof of Theorem 4.2 in [27], where one assumption is weaker and one is somewhat stronger than in the present paper. Here, we actually provide a different, simplified proof with $\operatorname{dim}(\Gamma)$ an arbitrary finite positive integer. Even this simplified proof requires substantial preparation. We begin as 
follows. Denote by $\mathbb{N}_{0}$ the set $\mathbb{N} \cup\{0\}$. Let $d \geq 1$. For every $\gamma_{0}=\left(\gamma_{1}^{0}, \gamma_{2}^{0}, \ldots, \gamma_{d}^{0}\right) \in \mathbb{C}^{d}$, denote by $\mathbb{C}^{d} F\left(\gamma_{0}\right)$ the real vector space of all formal power series

$$
f\left(\gamma_{1}, \gamma_{2}, \ldots, \gamma_{d}\right)=\sum_{\alpha \in \mathbb{N}_{0}^{d}} f_{\alpha}\left(\gamma_{1}-\gamma_{1}^{0}\right)^{\alpha_{1}}\left(\gamma_{2}-\gamma_{2}^{0}\right)^{\alpha_{2}} \ldots\left(\gamma_{d}-\gamma_{d}^{0}\right)^{\alpha_{d}},\left(\gamma_{1}, \gamma_{2}, \ldots, \gamma_{d}\right) \in \mathbb{C}^{d}
$$

with complex coefficients $f_{\alpha}$. Similarly, for every $\gamma_{0} \in \mathbb{R}^{d}$, denote by $\mathbb{R}^{d} F\left(\gamma_{0}\right)$ the real vector space of all formal power series

$$
g\left(\gamma_{1}, \gamma_{2}, \ldots, \gamma_{d}\right)=\sum_{\alpha \in \mathbb{N}_{0}^{d}} g_{\alpha}\left(\gamma_{1}-\gamma_{1}^{0}\right)^{\alpha_{1}}\left(\gamma_{2}-\gamma_{2}^{0}\right)^{\alpha_{2}} \ldots\left(\gamma_{d}-\gamma_{d}^{0}\right)^{\alpha_{d}},\left(\gamma_{1}, \gamma_{2}, \ldots, \gamma_{d}\right) \in \mathbb{R}^{d}
$$

with real coefficients $g_{\alpha}$. Moreover, for every $\gamma_{0} \in \mathbb{R}^{d}$, let $U_{d}^{\gamma_{0}}: \mathbb{R}^{d} F\left(\gamma_{0}\right) \rightarrow \mathbb{C}^{d} F\left(\gamma_{0}\right)$ be the linear operator which ascribes to each formal power series $\sum_{\alpha \in \mathbb{N}_{0}^{d}} g_{\alpha}\left(\gamma_{1}-\gamma_{1}^{0}\right)^{\alpha_{1}}\left(\gamma_{2}-\right.$ $\left.\gamma_{2}^{0}\right)^{\alpha_{2}} \ldots\left(\gamma_{d}-\gamma_{d}^{0}\right)^{\alpha_{d}}$ in $\mathbb{R}^{d} F\left(\gamma_{0}\right)$ the formal power series $\sum_{\alpha \in \mathbb{N}_{0}^{d}} g_{\alpha}\left(\gamma_{1}-\gamma_{1}^{0}\right)^{\alpha_{1}}\left(\gamma_{2}-\gamma_{2}^{0}\right)^{\alpha_{2}} \ldots\left(\gamma_{d}-\right.$ $\left.\gamma_{d}^{0}\right)^{\alpha_{d}}$ in $\mathbb{C}^{d} F\left(\gamma_{0}\right)$. Furthermore, for every $\gamma_{0} \in \mathbb{C}^{d}$, set

$$
I\left(\gamma_{0}\right)=\left(\operatorname{Re}\left(\gamma_{1}^{0}\right), \operatorname{Im}\left(\gamma_{1}^{0}\right), \operatorname{Re}\left(\gamma_{2}^{0}\right), \operatorname{Im}\left(\gamma_{2}^{0}\right), \ldots, \operatorname{Re}\left(\gamma_{d}^{0}\right), \operatorname{Im}\left(\gamma_{d}^{0}\right)\right) \in \mathbb{R}^{2 d},
$$

and let $\operatorname{Re}_{d}^{\gamma_{0}}: \mathbb{C}^{d} F\left(\gamma_{0}\right) \rightarrow \mathbb{R}^{2 d} F\left(I\left(\gamma_{0}\right)\right)$ be the operator which ascribes to every formal power series

$$
f\left(\gamma_{1}, \gamma_{2}, \ldots, \gamma_{d}\right)=\sum_{\alpha \in \mathbb{N}_{0}^{d}} f_{\alpha}\left(\gamma_{1}-\gamma_{1}^{0}\right)^{\alpha_{1}}\left(\gamma_{2}-\gamma_{2}^{0}\right)^{\alpha_{2}} \ldots\left(\gamma_{d}-\gamma_{d}^{0}\right)^{\alpha_{d}},\left(\gamma_{1}, \gamma_{2}, \ldots, \gamma_{d}\right) \in \mathbb{C}^{d},
$$

in $\mathbb{C}^{d} F\left(\gamma_{0}\right)$ the formal power series $\operatorname{Re}_{d}^{\gamma_{0}}(f) \in \mathbb{R}^{2 d} F\left(I\left(\gamma_{0}\right)\right)$ which is given by

$$
\operatorname{Re}_{d}^{\gamma_{0}}(f)\left(x_{1}, y_{1}, x_{2}, y_{2}, \ldots, x_{d}, y_{d}\right)=\sum_{\beta \in \mathbb{N}_{0}^{2 d}} c_{\beta} \prod_{j=1}^{d}\left(x_{j}-\operatorname{Re}\left(\gamma_{j}^{0}\right)\right)^{\beta_{j}^{(1)}}\left(y_{j}-\operatorname{Im}\left(\gamma_{j}^{0}\right)\right)^{\beta_{j}^{(2)}}
$$

where each $\beta \in \mathbb{N}_{0}^{2 d}$ is written in the form $\left(\beta_{1}^{(1)}, \beta_{1}^{(2)}, \beta_{2}^{(1)}, \beta_{2}^{(2)}, \ldots, \beta_{d}^{(1)}, \beta_{d}^{(2)}\right)$ and

$$
c_{\beta}=\operatorname{Re}\left(f_{\hat{\beta}} \prod_{j=1}^{d}\left(\begin{array}{c}
\beta_{j}^{(1)}+\beta_{j}^{(2)} \\
\beta_{j}^{(1)}
\end{array}\right) i^{\beta_{j}^{(2)}}\right)
$$

with $\hat{\beta}=\left(\beta_{1}^{(1)}+\beta_{1}^{(2)}, \beta_{2}^{(1)}+\beta_{2}^{(2)}, \ldots, \beta_{d}^{(1)}+\beta_{d}^{(2)}\right) \in \mathbb{N}_{0}^{d}$. Clearly, $f \mapsto \operatorname{Re}_{d}^{\gamma_{0}}(f)$ is a linear operator from the real vector space $\mathbb{C}^{d} F\left(\gamma_{0}\right)$ to the real vector space $\mathbb{R}^{2 d} F\left(I\left(\gamma_{0}\right)\right)$. Now, fix $R>0$ and consider $\operatorname{Hol}_{b}^{d}\left(D_{d}\left(\gamma_{0}, R\right)\right)$, the real vector space of all formal power series in $\mathbb{C}^{d} F\left(\gamma_{0}\right)$ that converge uniformly on the $d$-dimensional disk $D_{d}\left(\gamma_{0}, R\right)$. These series have finite supremum norms over $D_{d}\left(\gamma_{0}, R\right)$. Moreover, $\operatorname{Hol}_{b}^{d}\left(D_{d}\left(\gamma_{0}, R\right)\right)$ endowed with the supremum norm over $D_{d}\left(\gamma_{0}, R\right)$ is a normed vector space. Let

$$
\mathcal{R}_{d}^{\gamma_{0}}=U_{2 d}^{I\left(\gamma_{0}\right)} \circ \operatorname{Re}_{d}^{\gamma_{0}}: \mathbb{C}^{d} F\left(\gamma_{0}\right) \rightarrow \mathbb{C}^{2 d} F\left(I\left(\gamma_{0}\right)\right)
$$

We will need the following fact. This fact was proved in [15] as Lemma 8.1. 
Lemma 4.11. For every $\gamma_{0} \in \mathbb{C}^{d}$ and every $R>0$ we have

$$
\mathcal{R}_{d}^{\gamma_{0}}\left(\operatorname{Hol}_{b}^{d}\left(D_{d}\left(\gamma_{0}, R\right)\right)\right) \subset \operatorname{Hol}_{b}^{2 d}\left(D_{2 d}\left(I\left(\gamma_{0}\right), R / 4\right)\right) .
$$

Moreover, the linear operator $\mathcal{R}_{d}^{\gamma_{0}}: \operatorname{Hol}_{b}^{d}\left(D_{d}\left(\gamma_{0}, R\right)\right) \rightarrow \operatorname{Hol}_{b}^{2 d}\left(D_{2 d}\left(I\left(\gamma_{0}\right), R / 4\right)\right)$ is bounded and its norm is bounded above by $4^{d}$. Furthermore, for every $f \in \operatorname{Hol}_{b}^{d}\left(D_{d}\left(\gamma_{0}, R\right)\right)$,

$$
\left.\mathcal{R}_{d}^{\gamma_{0}}(f)\right|_{D_{d}\left(\gamma_{0}, R / 4\right)}=\left.\operatorname{Re}(f)\right|_{D_{d}\left(\gamma_{0}, R / 4\right)},
$$

where $\operatorname{Re}(f)$ is the real part of the complex-valued function $f$ and $D_{d}\left(\gamma_{0}, R / 4\right)$ is identified with $D_{2 d}\left(I\left(\gamma_{0}\right), R / 4\right) \cap \mathbb{R}^{2 d}$ as $\mathbb{C}^{d}$ is embedded in $\mathbb{C}^{2 d}$ by the formula $\left(x_{1}+i y_{1}, x_{2}+i y_{2}, \ldots, x_{d}+i y_{d}\right) \mapsto$ $\left(x_{1}, y_{1}, x_{2}, y_{2}, \ldots, x_{d}, y_{d}\right)$.

Passing to other ingredients needed in our proof of Theorem 4.10, we recall the space of Hölder continuous functions. Let $\alpha>0$. We say that a function $g: I^{\infty} \rightarrow \mathbb{C}$ is $\alpha$-Hölder continuous if

$$
v_{\alpha}(g):=\sup _{n \in \mathbb{N}}\left\{v_{\alpha, n}(g)\right\}<\infty
$$

where

$$
v_{\alpha, n}(g)=\sup \left\{|g(\omega)-g(\tau)| e^{\alpha n}: \omega, \tau \in I^{\infty} \text { and }|\omega \wedge \tau| \geq n\right\} .
$$

Let $\mathcal{K}_{\alpha}$ be the set of all complex-valued $\alpha$-Hölder continuous (but not necessarily bounded) functions defined on $I^{\infty}$. Set

$$
\mathcal{K}_{\alpha}^{S}:=\left\{u \in \mathcal{K}_{\alpha}: \sum_{i \in I} \exp \left(\sup _{\omega \in[i]} \operatorname{Re}(u(\omega))\right)<\infty\right\} .
$$

Members of $\mathcal{K}_{\alpha}^{S}$ are called $\alpha$-Hölder summable potentials. If a function $g: I^{\infty} \rightarrow \mathbb{C}$ has finite supremum norm $\|g\|_{\infty}$ and finite $v_{\alpha}(g)$, then $g$ is said to belong to the space $\mathcal{H}_{\alpha}$ of all bounded $\alpha$-Hölder continuous functions defined on $I^{\infty}$. $\mathcal{H}_{\alpha}$ becomes a Banach space when equipped with the norm $\|\cdot\|_{\alpha}$ given by the formula

$$
\|g\|_{\alpha}=\|g\|_{\infty}+v_{\alpha}(g)
$$

Lemma 4.12. For every $g, h \in \mathcal{H}_{\alpha}$ we have $\|g h\|_{\alpha} \leq 3\|g\|_{\alpha}\|h\|_{\alpha}$.

Proof. Indeed, $\|g h\|_{\infty} \leq\|g\|_{\infty}\|h\|_{\infty} \leq\|g\|_{\alpha}\|h\|_{\alpha}$. Moreover, for every $\omega, \tau \in I^{\infty}$ such that $|\omega \wedge \tau| \geq 1$ we have

$$
\begin{aligned}
|(g h)(\omega)-(g h)(\tau)| & \leq|g(\omega)||h(\omega)-h(\tau)|+|h(\tau)||g(\omega)-g(\tau)| \\
& \leq\|g\|_{\infty} v_{\alpha}(h) e^{-\alpha|\omega \wedge \tau|}+\|h\|_{\infty} v_{\alpha}(g) e^{-\alpha|\omega \wedge \tau|} \\
& \leq 2\|g\|_{\alpha}\|h\|_{\alpha} e^{-\alpha|\omega \wedge \tau|}
\end{aligned}
$$

that is, $v_{\alpha}(g h) \leq 2\|g\|_{\alpha}\|h\|_{\alpha}$. Consequently, $\|g h\|_{\alpha} \leq 3\|g\|_{\alpha}\|h\|_{\alpha}$. 
In particular, this lemma shows that $\mathcal{H}_{\alpha}$ becomes a Banach algebra if one replaces $\|\cdot\|_{\alpha}$ by an equivalent norm $\|\cdot\|_{\alpha}^{\prime}$ such that $\|g h\|_{\alpha}^{\prime} \leq\|g\|_{\alpha}^{\prime}\|h\|_{\alpha}^{\prime}$. The factors "3" and "9" in the following lemma would then "disappear".

Lemma 4.13. For every $g \in \mathcal{H}_{\alpha}$,

$$
\left\|e^{g}\right\|_{\alpha} \leq e^{3\|g\|_{\alpha}} \quad \text { and } \quad\left\|e^{g}-1\right\|_{\alpha} \leq 3\|g\|_{\alpha} e^{3\|g\|_{\alpha}}
$$

and for every $g, h \in \mathcal{H}_{\alpha}$,

$$
\left\|e^{h}-e^{g}\right\|_{\alpha} \leq 9 e^{3\left(\|g\|_{\alpha}+\|h-g\|_{\alpha}\right)}\|h-g\|_{\alpha} .
$$

In particular, the map $g \in \mathcal{H}_{\alpha} \mapsto e^{g} \in \mathcal{H}_{\alpha}$ is continuous.

Proof. Since $e^{g}=\sum_{n=0}^{\infty} g^{n} / n$ !, it follows from Lemma 4.12 that $\left\|e^{g}\right\|_{\alpha} \leq e^{3\|g\|_{\alpha}}$. Moreover,

$$
\begin{aligned}
\left\|e^{g}-1\right\|_{\alpha} & =\left\|\sum_{n=1}^{\infty} \frac{g^{n}}{n !}\right\|_{\alpha}=\left\|g \sum_{n=0}^{\infty} \frac{g^{n}}{(n+1) !}\right\|_{\alpha} \\
& \leq 3\|g\|_{\alpha}\left\|\sum_{n=0}^{\infty} \frac{g^{n}}{(n+1) !}\right\|_{\alpha} \leq 3\|g\|_{\alpha} \sum_{n=0}^{\infty} \frac{\left\|g^{n}\right\|_{\alpha}}{(n+1) !} \\
& \leq 3\|g\|_{\alpha} \sum_{n=0}^{\infty} \frac{\left(3\|g\|_{\alpha}\right)^{n}}{(n+1) !} \leq 3\|g\|_{\alpha} \sum_{n=0}^{\infty} \frac{\left(3\|g\|_{\alpha}\right)^{n}}{n !}=3\|g\|_{\alpha} e^{3\|g\|_{\alpha}} .
\end{aligned}
$$

Hence, using Lemma 4.12 and the above two estimates, we deduce that for every $g, h \in \mathcal{H}_{\alpha}$,

$$
\left\|e^{h}-e^{g}\right\|_{\alpha}=\left\|e^{g}\left(e^{h-g}-1\right)\right\|_{\alpha} \leq 3\left\|e^{g}\right\|_{\alpha}\left\|e^{h-g}-1\right\|_{\alpha} \leq 9 e^{3\left(\|g\|_{\alpha}+\|h-g\|_{\alpha}\right)}\|h-g\|_{\alpha} .
$$

Now, for every $u \in \mathcal{K}_{\alpha}^{S}$ and $g \in \mathcal{H}_{\alpha}$ define

$$
\mathcal{L}_{u} g(\omega)=\sum_{i \in I} e^{u(i \omega)} g(i \omega)
$$

Easy calculations show that $\mathcal{L}_{u} g \in \mathcal{H}_{\alpha}$ and that the operator $\mathcal{L}_{u}: \mathcal{H}_{\alpha} \rightarrow \mathcal{H}_{\alpha}$ is linear and bounded. We hence have the following.

Lemma 4.14. If $u \in \mathcal{K}_{\alpha}^{S}$, then $\mathcal{L}_{u} \in L\left(\mathcal{H}_{\alpha}\right)$, where $L\left(\mathcal{H}_{\alpha}\right)$ denotes the Banach space of all bounded, linear operators on $\mathcal{H}_{\alpha}$, endowed with the operator norm.

We immediately obtain the following.

Corollary 4.15. Let $\Phi \in \operatorname{HIFS}(X)$ and $s \in(0,1)$ be a contraction ratio for $\Phi$. Let $0<\alpha \leq$ $-\log s$. If $P(\Phi, t)<\infty$ and $g \in \mathcal{H}_{\alpha}$, then the potential $u(\omega)=g(\omega)+t \log \left|\varphi_{\omega_{1}}^{\prime}\left(\pi_{\Phi}(\sigma(\omega))\right)\right|$ belongs to $\mathcal{K}_{\alpha}^{S}$ and the corresponding linear operator $\mathcal{L}_{u}: \mathcal{H}_{\alpha} \rightarrow \mathcal{H}_{\alpha}$ is bounded. 
Proof. We simply need to prove that $u \in \mathcal{K}_{\alpha}^{S}$. To do this, observe that by Remark 2.1 and Lemma 2.2 , for all $\omega, \tau \in I^{\infty}$ with $|\omega \wedge \tau| \geq 1$ we have

$$
\begin{aligned}
|\log | \varphi_{\omega_{1}}^{\prime}\left(\pi_{\Phi}(\sigma(\omega))\right)|-\log | \varphi_{\tau_{1}}^{\prime}\left(\pi_{\Phi}(\sigma(\tau))\right)|| & \leq L(1-s)^{-1}\left|\pi_{\Phi}(\sigma(\omega))-\pi_{\Phi}(\sigma(\tau))\right| \\
& \leq L(1-s)^{-1} e^{-\alpha(|\omega \wedge \tau|-1)} \operatorname{diam}(X) .
\end{aligned}
$$

Thus, the function $\omega \mapsto \log \left|\varphi_{\omega_{1}}^{\prime}\left(\pi_{\Phi}(\sigma(\omega))\right)\right|$ is in $\mathcal{K}_{\alpha}$, as $v_{\alpha}\left(\omega \mapsto \log \left|\varphi_{\omega_{1}}^{\prime}\left(\pi_{\Phi}(\sigma(\omega))\right)\right|\right) \leq$ $L(1-s)^{-1} e^{\alpha} \operatorname{diam}(X)$. Since $g \in \mathcal{K}_{\alpha}$ as well, we conclude that $u \in \mathcal{K}_{\alpha}$. Moreover,

$$
\begin{aligned}
\sum_{i \in I} \exp \left(\sup _{\omega \in[i]} \operatorname{Re}(u(\omega))\right) & =\sum_{i \in I} \exp \left(\sup _{\omega \in[i]} \operatorname{Re}\left(g(\omega)+t \log \left|\varphi_{i}^{\prime}\left(\pi_{\Phi}(\sigma(\omega))\right)\right|\right)\right) \\
& \leq \sum_{i \in I} \exp \left(\sup _{\omega \in[i]} \operatorname{Re}(g(\omega))\right)\left\|\varphi_{i}^{\prime}\right\|^{t} \\
& \leq e^{\|g\|_{\infty}} \sum_{i \in I}\left\|\varphi_{i}^{\prime}\right\|^{t}<\infty
\end{aligned}
$$

since $P(\Phi, t)<\infty$. Thus, $u$ is summable.

We shall now prove the following result. Note that the finite-dimensional complex manifold $\tilde{\Gamma}$ in this result is completely general. It need not be the same and is generally not the same as the manifold $\Gamma$ from Theorem 4.10. This important and necessary distinction will become clearer in Lemma 4.23 , where $\Gamma$ will be the manifold from Theorem 4.10 and is a subset of $\mathbb{C}^{d}$, whereas $\tilde{\Gamma}$ will be $\mathbb{C}^{2 d}$. Thus, in our case, $\Gamma$ can be naturally embedded in $\tilde{\Gamma}$ via the embedding of $\mathbb{C}^{d}$ in $\mathbb{C}^{2 d}$ given by the formula $\left(x_{1}+i y_{1}, x_{2}+i y_{2}, \ldots, x_{d}+i y_{d}\right) \mapsto\left(x_{1}, y_{1}, x_{2}, y_{2}, \ldots, x_{d}, y_{d}\right)$.

Lemma 4.16. Let $G$ be an open subset of a finite-dimensional complex manifold $\tilde{\Gamma}$. Let $\tilde{\gamma}_{0} \in G$ and $\Phi^{\tilde{\gamma}_{0}} \in \operatorname{HIFS}(X)$. Let also $t \geq 0$ be such that $P\left(\tilde{\gamma}_{0}, t\right)<\infty$, and $s \in(0,1)$ a contraction ratio for $\Phi^{\tilde{\gamma}_{0}}$. Set $0<\alpha \leq-\log s$. Let $\tilde{\gamma} \mapsto g_{\tilde{\gamma}} \in \mathcal{H}_{\alpha}$ be a function with the following properties:

(a) $\sup \left\{\left\|g_{\tilde{\gamma}}\right\|_{\alpha}: \tilde{\gamma} \in G\right\}<\infty$; and

(b) for every $\omega \in I^{\infty}$ the function $\tilde{\gamma} \mapsto g_{\tilde{\gamma}}(\omega)$ from $G$ to $\mathbb{C}$ is holomorphic.

Set $\mathcal{L}_{\tilde{\gamma}}:=\mathcal{L}_{u_{\tilde{\gamma}}}$, where $u_{\tilde{\gamma}}(\omega)=g_{\tilde{\gamma}}(\omega)+t \log \left|\left(\varphi_{\omega_{1}}^{\tilde{\gamma}_{0}}\right)^{\prime}\left(\pi_{\tilde{\gamma}_{0}}(\sigma(\omega))\right)\right|$. Then the map $\tilde{\gamma} \mapsto \mathcal{L}_{\tilde{\gamma}} \in$ $L\left(\mathcal{H}_{\alpha}\right)$ is holomorphic.

In order to prove this result, we need a few preparatory lemmas. Moreover, in order to allege notation, we use $\gamma$ instead of $\tilde{\gamma}$ from this point on until the end of the proof of Lemma 4.16. Also, observe that

$$
\mathcal{L}_{\gamma} f(\omega)=\sum_{i \in I} e^{u_{\gamma}(i \omega)} f(i \omega)=\sum_{i \in I} e^{g_{\gamma}(i \omega)}\left|\left(\varphi_{i}^{\gamma_{0}}\right)^{\prime}\left(\pi_{\gamma_{0}}(\omega)\right)\right|^{t} f(i \omega)
$$

for every $\omega \in I^{\infty}$. For every $i \in I$ and $\gamma \in G$, we define the linear operator $\mathcal{L}_{\gamma, i}: \mathcal{H}_{\alpha} \rightarrow \mathcal{H}_{\alpha}$ as

$$
\mathcal{L}_{\gamma, i} f(\omega)=e^{u_{\gamma}(i \omega)} f(i \omega)=e^{g_{\gamma}(i \omega)}\left|\left(\varphi_{i}^{\gamma_{0}}\right)^{\prime}\left(\pi_{\gamma_{0}}(\omega)\right)\right|^{t} f(i \omega) .
$$


Finally, we let $H:=\sup \left\{\left\|g_{\bar{\gamma}}\right\|_{\alpha}: \bar{\gamma} \in G\right\}$.

Lemma 4.17. Under the assumptions of Lemma 4.16, the function $\gamma \mapsto g_{\gamma}$ from $G$ to $\mathcal{H}_{\alpha}$ is continuous.

Proof. Applying Cauchy's formula to the holomorphic functions $\gamma \mapsto g_{\gamma}(\omega), \omega \in I^{\infty}$, we obtain that for each $\zeta \in G$ and each $\varepsilon>0$, there exists a $\delta_{1}>0$ such that for each $\gamma \in G$ with $|\gamma-\zeta|<\delta_{1}$ and for all $\omega \in I^{\infty}$,

$$
\left|g_{\gamma}(\omega)-g_{\zeta}(\omega)\right|<\varepsilon H .
$$

Hence for each $\gamma \in G$ with $|\gamma-\zeta|<\delta_{1}$,

$$
\left\|g_{\gamma}-g_{\zeta}\right\|_{\infty}<\varepsilon H
$$

Moreover, for every $\gamma, \xi, \zeta \in G$ and for all $\omega, \tau \in I^{\infty}$ such that $|\omega \wedge \tau| \geq 1$,

$$
\left|\left(g_{\gamma}(\omega)-g_{\zeta}(\omega)\right)-\left(g_{\gamma}(\tau)-g_{\zeta}(\tau)\right)\right|=\left|\left(g_{\gamma}(\omega)-g_{\gamma}(\tau)\right)-\left(g_{\zeta}(\omega)-g_{\zeta}(\tau)\right)\right|
$$

and

$$
\left|g_{\xi}(\omega)-g_{\xi}(\tau)\right| \leq H e^{-\alpha|\omega \wedge \tau|} .
$$

Applying Cauchy's formula to the holomorphic functions $\gamma \mapsto g_{\gamma}(\omega)-g_{\gamma}(\tau), \omega, \tau \in I^{\infty}$, and using the previous two relations and condition (a), we obtain that for each $\zeta \in G$ and each $\varepsilon>0$, there exists a $\delta_{2}>0$ such that for each $\gamma \in G$ with $|\gamma-\zeta|<\delta_{2}$ and for all $\omega, \tau \in I^{\infty}$ such that $|\omega \wedge \tau| \geq 1$,

$$
\left|\left(g_{\gamma}(\omega)-g_{\zeta}(\omega)\right)-\left(g_{\gamma}(\tau)-g_{\zeta}(\tau)\right)\right| \leq \varepsilon H e^{-\alpha|\omega \wedge \tau|}
$$

Hence for each $\gamma \in G$ with $|\gamma-\zeta|<\delta_{2}$,

$$
v_{\alpha}\left(g_{\gamma}-g_{\zeta}\right) \leq \varepsilon H
$$

From (4.1) and (4.2), it follows that for each $\gamma \in G$ with $|\gamma-\zeta|<\min \left\{\delta_{1}, \delta_{2}\right\}$,

$$
\left\|g_{\gamma}-g_{\zeta}\right\|_{\alpha} \leq 2 \varepsilon H
$$

Therefore, the function $\gamma \mapsto g_{\gamma}$ is continuous.

Lemma 4.18. $\left\|\left|\left(\varphi_{i}^{\gamma_{0}}\right)^{\prime} \circ \pi_{\gamma_{0}}\right|^{t}\right\|_{\alpha} \leq C\left\|\left(\varphi_{i}^{\gamma_{0}}\right)^{\prime}\right\|^{t}$, where $C=1+K \operatorname{Ltdiam}(X)$.

Proof. In view of conditions (iv) and (iv') defining CIFSs in section 2, we get for all $\omega, \tau \in I^{\infty}$ with $|\omega \wedge \tau| \geq 1$ that

$$
\begin{aligned}
\left.||\left(\varphi_{i}^{\gamma_{0}}\right)^{\prime}\left(\pi_{\gamma_{0}}(\omega)\right)\right|^{t}-\left|\left(\varphi_{i}^{\gamma_{0}}\right)^{\prime}\left(\pi_{\gamma_{0}}(\tau)\right)\right|^{t} \mid & \leq t\left\|\left(\varphi_{i}^{\gamma_{0}}\right)^{\prime}\right\|^{t-1}||\left(\varphi_{i}^{\gamma_{0}}\right)^{\prime}\left(\pi_{\gamma_{0}}(\omega)\right)|-|\left(\varphi_{i}^{\gamma_{0}}\right)^{\prime}\left(\pi_{\gamma_{0}}(\tau)\right)|| \\
& \leq t\left\|\left(\varphi_{i}^{\gamma_{0}}\right)^{\prime}\right\|^{t-1} L\left\|\left[\left(\varphi_{i}^{\gamma_{0}}\right)^{\prime}\right]^{-1}\right\|_{V}^{-1}\left|\pi_{\gamma_{0}}(\omega)-\pi_{\gamma_{0}}(\tau)\right| \\
& \leq t\left\|\left(\varphi_{i}^{\gamma_{0}}\right)^{\prime}\right\|^{t-1} L K\left\|\left(\varphi_{i}^{\gamma_{0}}\right)^{\prime}\right\| s^{|\omega \wedge \tau|} \operatorname{diam}(X) \\
& \leq K L t\left\|\left(\varphi_{i}^{\gamma_{0}}\right)^{\prime}\right\|^{t} e^{-\alpha|\omega \wedge \tau|} \operatorname{diam}(X) \\
& \leq K \operatorname{Lidiam}(X)\left\|\left(\varphi_{i}^{\gamma_{0}}\right)^{\prime}\right\|^{t} e^{-\alpha|\omega \wedge \tau|} .
\end{aligned}
$$


Therefore $v_{\alpha}\left(\left|\left(\varphi_{i}^{\gamma_{0}}\right)^{\prime} \circ \pi_{\gamma_{0}}\right|^{t}\right) \leq K L t \operatorname{diam}(X)\left\|\left(\varphi_{i}^{\gamma_{0}}\right)^{\prime}\right\|^{t}$. It is then straightforward to see that $\left\|\left|\left(\varphi_{i}^{\gamma_{0}}\right)^{\prime} \circ \pi_{\gamma_{0}}\right|^{t}\right\|_{\alpha} \leq(1+K \operatorname{Lt} \operatorname{diam}(X))\left\|\left(\varphi_{i}^{\gamma_{0}}\right)^{\prime}\right\|^{t}$.

Lemma 4.19. Under the assumptions of Lemma 4.16, for each $i \in I$ the function $\gamma \mapsto \mathcal{L}_{\gamma, i}$ from $G$ to $L\left(\mathcal{H}_{\alpha}\right)$ is continuous. Moreover, $\left\|\mathcal{L}_{\gamma, i}\right\| \leq 9 e^{3 H} C\left\|\left(\varphi_{i}^{\gamma_{0}}\right)^{\prime}\right\|^{t}$.

Proof. From the expression of $\mathcal{L}_{\gamma, i} f$ and Lemmas 4.12, 4.13 and 4.18, we deduce that for every $\gamma, \zeta \in G$ and for every $f \in \mathcal{H}_{\alpha}$,

$$
\begin{aligned}
\left\|\mathcal{L}_{\gamma, i} f-\mathcal{L}_{\zeta, i} f\right\|_{\alpha} & \leq 9\left\|e^{g_{\gamma}}-e^{g_{\zeta}}\right\|_{\alpha}\left\|\left.\left(\varphi_{i}^{\gamma_{0}}\right)^{\prime} \circ \pi_{\gamma_{0}}\right|^{t}\right\|_{\alpha}\|f\|_{\alpha} \\
& \leq 9 \cdot 9 e^{3\left(\left\|g_{\gamma}\right\|_{\alpha}+\left\|g_{\gamma}-g_{\zeta}\right\|_{\alpha}\right)}\left\|g_{\gamma}-g_{\zeta}\right\|_{\alpha} \cdot C\left\|\left(\varphi_{i}^{\gamma_{0}}\right)^{\prime}\right\|^{t} \cdot\|f\|_{\alpha} \\
& \leq 81 C\left\|\left(\varphi_{i}^{\gamma_{0}}\right)^{\prime}\right\|^{t} e^{3\left(\left\|g_{\gamma}\right\|_{\alpha}+\left\|g_{\gamma}-g_{\zeta}\right\|_{\alpha}\right)}\left\|g_{\gamma}-g_{\zeta}\right\|_{\alpha}\|f\|_{\alpha} .
\end{aligned}
$$

Therefore $\left\|\mathcal{L}_{\gamma, i}-\mathcal{L}_{\zeta, i}\right\| \leq 81 C\left\|\left(\varphi_{i}^{\gamma_{0}}\right)^{\prime}\right\|{ }^{t} e^{3\left(\left\|g_{\gamma}\right\|_{\alpha}+\left\|g_{\gamma}-g_{\zeta}\right\|_{\alpha}\right)}\left\|g_{\gamma}-g_{\zeta}\right\|_{\alpha}$ and the continuity of $\gamma \mapsto$ $\mathcal{L}_{\gamma, i}$ follows from that of $\gamma \mapsto g_{\gamma}$, that is, from Lemma 4.17 .

Furthermore, from the expression of $\mathcal{L}_{\gamma, i} f$ and Lemmas 4.12, 4.13 and 4.18, we obtain that

$$
\begin{aligned}
\left\|\mathcal{L}_{\gamma, i} f\right\|_{\alpha} & \leq 9\left\|e^{g_{\gamma}}\right\|_{\alpha}\left\|\left|\left(\varphi_{i}^{\gamma_{0}}\right)^{\prime} \circ \pi_{\gamma_{0}}\right|^{t}\right\|_{\alpha}\|f\|_{\alpha} \\
& \leq 9 e^{3\left\|g_{\gamma}\right\|_{\alpha}} \cdot C\left\|\left(\varphi_{i}^{\gamma_{0}}\right)^{\prime}\right\|^{t} \cdot\|f\|_{\alpha} \\
& \leq 9 e^{3 H} C\left\|\left(\varphi_{i}^{\gamma_{0}}\right)^{\prime}\right\|^{t}\|f\|_{\alpha} .
\end{aligned}
$$

Thus, $\left\|\mathcal{L}_{\gamma, i}\right\| \leq 9 e^{3 H} C\left\|\left(\varphi_{i}^{\gamma_{0}}\right)^{\prime}\right\|^{t}$

We now prove the holomorphicity of that function.

Lemma 4.20. Under the assumptions of Lemma 4.16, for each $i \in I$ the function $\gamma \mapsto \mathcal{L}_{\gamma, i}$ from $G$ to $L\left(\mathcal{H}_{\alpha}\right)$ is holomorphic.

Proof. Indeed, from Lemma 4.19 and Osgood's Lemma for (Banach space)-valued functions (see the argument on page 3 of [6]), we may assume that $\operatorname{dim}_{\mathbb{C}} \tilde{\Gamma}=1$. Fix $i \in I$. Let $f \in \mathcal{H}_{\alpha}$, $\omega \in I^{\infty}$ and let $T$ be any triangle in $G$. Since the function $\gamma \mapsto \mathcal{L}_{\gamma, i} f(\omega)$ from $G$ to $\mathbb{C}$ is holomorphic by condition (b) of Lemma 4.16, we have that

Therefore

$$
\int_{\partial T} \mathcal{L}_{\gamma, i} f(\omega) d \gamma=0
$$

$$
\left(\int_{\partial T} \mathcal{L}_{\gamma, i} d \gamma\right)(f)(\omega)=\int_{\partial T} \mathcal{L}_{\gamma, i} f(\omega) d \gamma=0 .
$$

Since this holds for all $\omega \in I^{\infty}$ and for all $f \in \mathcal{H}_{\alpha}$, we deduce that

$$
\int_{\partial T} \mathcal{L}_{\gamma, i} d \gamma=0
$$

As the triangle $T$ was chosen arbitrarily in $G$, we conclude from Morera's Theorem that the function $\gamma \mapsto \mathcal{L}_{\gamma, i}$ from $G$ to $L\left(\mathcal{H}_{\alpha}\right)$ is holomorphic. 
Proof of Lemma 4.16. Since $\mathcal{L}_{\gamma}=\sum_{i \in I} \mathcal{L}_{\gamma, i}$, since all the functions $\gamma \mapsto \mathcal{L}_{\gamma, i}$ are holomorphic according to Lemma 4.20, since $\left\|\mathcal{L}_{\gamma, i}\right\| \leq 9 e^{3 H} C\left\|\left(\varphi_{i}^{\gamma_{0}}\right)^{\prime}\right\|^{t}$ for every $i \in I$ according to Lemma 4.19, since the series $\sum_{i \in I}\left\|\left(\varphi_{i}^{\gamma_{0}}\right)^{\prime}\right\|^{t}$ converges as $P\left(\gamma_{0}, t\right)<\infty$, and since the sum of a uniformly convergent sequence of holomorphic functions is holomorphic, we deduce that the function $\gamma \mapsto \mathcal{L}_{\gamma}$ from $G$ to $L\left(\mathcal{H}_{\alpha}\right)$ is holomorphic.

We continue our preparation by looking at the coding map.

Lemma 4.21. Let $\left\{\Phi^{\gamma}\right\}_{\gamma \in \Gamma}$ be an analytic family in $\operatorname{HIFS}(X)$. For any fixed $\omega \in I^{\infty}$, the function $\gamma \mapsto \pi_{\gamma}(\omega)$ is analytic and the family $\left\{\gamma \mapsto \pi_{\gamma}(\omega)\right\}_{\omega \in I^{\infty}}$ is equicontinuous on $\Gamma$.

Proof. Let $x \in X$. Fix for a moment $\omega \in I^{\infty}$. Then $\pi_{\gamma}(\omega)=\lim _{n \rightarrow \infty} \varphi_{\omega_{1}}^{\gamma} \circ \cdots \circ \varphi_{\omega_{n}}^{\gamma}(x)$. Hence, $\gamma \mapsto \pi_{\gamma}(\omega)$ is a limit of a sequence of uniformly bounded holomorphic maps defined on $\Gamma$. Since a family of uniformly bounded holomorphic maps is normal, it follows that $\gamma \mapsto \pi_{\gamma}(\omega)$ is holomorphic. Furthermore, $\left\{\gamma \mapsto \pi_{\gamma}(\omega)\right\}_{\omega \in I^{\infty}}$ is uniformly bounded. Hence, it is normal and thereby equicontinuous.

We now introduce a function $\kappa$ and describe some of its properties.

Lemma 4.22. In the setting of Theorem 4.10, fix $\gamma_{0} \in \Gamma$ and $R_{0}>0$ so that $D_{d}\left(\gamma_{0}, R_{0}\right) \subset \Gamma_{0}$. For every $\omega \in I^{\infty}$ define

$$
\kappa_{\omega}(\gamma)=\frac{\left(\varphi_{\omega_{1}}^{\gamma}\right)^{\prime}\left(\pi_{\gamma}(\sigma(\omega))\right)}{\left(\varphi_{\omega_{1}}^{\gamma_{0}}\right)^{\prime}\left(\pi_{\gamma_{0}}(\sigma(\omega))\right)}, \quad \gamma \in D_{d}\left(\gamma_{0}, R_{0}\right) .
$$

Then we have that

$$
\left|\kappa_{\omega}(\gamma)-1\right| \leq \frac{1}{4}
$$

for all $\omega \in I^{\infty}$ and all $\gamma \in D_{d}\left(\gamma_{0}, R_{0} / 8 B\right)$, and thus for all $\omega \in I^{\infty}$ there exists a unique holomorphic branch $\log \kappa_{\omega}$ of the logarithm of $\kappa_{\omega}$ defined on $D_{d}\left(\gamma_{0}, R_{0} / 8 B\right)$ and sending $\gamma_{0}$ to 0 . Furthermore, there exists a constant $M>0$ such that

$$
\left|\log \kappa_{\omega}(\gamma)\right| \leq M
$$

for all $\omega \in I^{\infty}$ and all $\gamma \in D_{d}\left(\gamma_{0}, R_{0} / 8 B\right)$.

Proof. According to Condition (1) from Theorem 4.10 we have that

$$
\left|\kappa_{\omega}(\gamma)\right| \leq B
$$

for all $\omega \in I^{\infty}$ and all $\gamma \in D_{d}\left(\gamma_{0}, R_{0}\right)$. Choosing an appropriate rotation to change the system of coordinates we may assume without loss of generality that the dimension $d=1$. Since for all $\gamma \in B\left(\gamma_{0}, R_{0} / 2\right)$ and all $0<r \leq R_{0} / 2$,

$$
\kappa_{\omega}^{\prime}(\gamma)=\frac{1}{2 \pi i} \int_{\partial B(\gamma, r)} \frac{\kappa_{\omega}(\xi)}{(\xi-\gamma)^{2}} d \xi
$$


we obtain from (4.3) that

$$
\left|\kappa_{\omega}^{\prime}(\gamma)\right| \leq \frac{1}{2 \pi} \int_{\partial B(\gamma, r)} \frac{B}{r^{2}}|d \xi|=\frac{B}{r} .
$$

Since $\kappa_{\omega}\left(\gamma_{0}\right)=1$, we therefore get for all $\gamma \in B\left(\gamma_{0}, r\right)$ that

$$
\left|\kappa_{\omega}(\gamma)-1\right|=\left|\kappa_{\omega}(\gamma)-\kappa_{\omega}\left(\gamma_{0}\right)\right|=\left|\int_{\gamma_{0}}^{\gamma} \kappa_{\omega}^{\prime}(\xi) d \xi\right| \leq \int_{\gamma_{0}}^{\gamma}\left|\kappa_{\omega}^{\prime}(\xi)\right||d \xi| \leq \frac{B}{r}\left|\gamma-\gamma_{0}\right| .
$$

Taking $r=R_{0} / 2$, we deduce that $\left|\kappa_{\omega}(\gamma)-1\right| \leq 1 / 4$ for all $\gamma \in D_{d}\left(\gamma_{0}, R_{0} / 8 B\right)$.

Our last auxiliary result is the following.

Lemma 4.23. In the setting of Theorem 4.10, fix $\gamma_{0} \in \Gamma$ and $R_{0}>0$ so that $D_{d}\left(\gamma_{0}, R_{0}\right) \subset \Gamma_{0}$. Let $s \in(0,1)$ be a contraction ratio for $\Phi^{\gamma_{0}}$. Set $0<\alpha<-\log s$ and choose $0<R<R_{0} / 8 B$ so small that $e^{-\alpha}$ is a contraction ratio for all $\gamma \in D_{d}\left(\gamma_{0}, R\right)$. Then the map $\tilde{\gamma} \mapsto \mathcal{L}_{\tilde{\gamma}} \in L\left(\mathcal{H}_{\alpha}\right)$, $\tilde{\gamma} \in D_{2 d}\left(I\left(\gamma_{0}\right), R / 4\right)$, is holomorphic, where $\mathcal{L}_{\tilde{\gamma}}:=\mathcal{L}_{u_{\tilde{\gamma}}}$ with

$$
u_{\tilde{\gamma}}(\omega)=t \mathcal{R}_{d}^{\gamma_{0}}\left(\log \kappa_{\omega}\right)(\tilde{\gamma})+t \log \left|\left(\varphi_{\omega_{1}}^{\gamma_{0}}\right)^{\prime}\left(\pi_{\gamma_{0}}(\sigma(\omega))\right)\right| .
$$

Proof. Let $\tilde{\Gamma}=\mathbb{C}^{2 d}, G=D_{2 d}\left(I\left(\gamma_{0}\right), R / 4\right), \tilde{\gamma}_{0}=I\left(\gamma_{0}\right), \Phi^{\tilde{\gamma}_{0}}=\Phi^{\gamma_{0}}$, and let $g_{\tilde{\gamma}}(\omega)=$ $t \mathcal{R}_{d}^{\gamma_{0}}\left(\log \kappa_{\omega}\right)(\tilde{\gamma})$. According to Lemma 4.16, it suffices to prove that for all $\tilde{\gamma} \in D_{2 d}\left(I\left(\gamma_{0}\right), R / 4\right)$ the function $\omega \mapsto \mathcal{R}_{d}^{\gamma_{0}}\left(\log \kappa_{\omega}\right)(\tilde{\gamma})$ belongs to $\mathcal{H}_{\alpha}$, and that the function $\tilde{\gamma} \mapsto \mathcal{R}_{d}^{\gamma_{0}}(\log \kappa)(\tilde{\gamma})$ from $D_{2 d}\left(I\left(\gamma_{0}\right), R / 4\right)$ to $\mathcal{H}_{\alpha}$ satisfies conditions (a) and (b). Condition (b) is clearly satisfied. Moreover, it follows from Lemmas 4.11 and 4.22 that

$$
\left\|\mathcal{R}_{d}^{\gamma_{0}}(\log \kappa)(\tilde{\gamma})\right\|_{\infty} \leq 4^{d} M,
$$

where $M$ is the constant arising from Lemma 4.22.

Claim: There exists a constant $C_{0}>0$ such that for all $\omega, \tau \in I^{\infty}$ and for all $\gamma \in D_{d}\left(\gamma_{0}, R\right)$,

$$
\left|\log \kappa_{\omega}(\gamma)-\log \kappa_{\tau}(\gamma)\right| \leq C_{0} e^{-\alpha|\omega \wedge \tau|} .
$$

In order to prove the above claim, observe that if $\omega_{1} \neq \tau_{1}$, then

$$
\left|\log \kappa_{\omega}(\gamma)-\log \kappa_{\tau}(\gamma)\right| \leq\left|\log \kappa_{\omega}(\gamma)\right|+\left|\log \kappa_{\tau}(\gamma)\right| \leq 2 M=2 M e^{-\alpha|\omega \wedge \tau|} .
$$

Thus, suppose $\omega_{1}=\tau_{1}=i$ for some $i \in I$. Let $\beta_{\gamma, \omega}:=\pi_{\gamma}(\sigma(\omega))$. Then

$$
\begin{aligned}
\mid \kappa_{\omega}(\gamma)- & \kappa_{\tau}(\gamma) \mid \\
= & \left|\frac{\left(\varphi_{i}^{\gamma}\right)^{\prime}\left(\pi_{\gamma}(\sigma(\omega))\right)\left(\varphi_{i}^{\gamma_{0}}\right)^{\prime}\left(\pi_{\gamma_{0}}(\sigma(\tau))\right)-\left(\varphi_{i}^{\gamma}\right)^{\prime}\left(\pi_{\gamma}(\sigma(\tau))\right)\left(\varphi_{i}^{\gamma_{0}}\right)^{\prime}\left(\pi_{\gamma_{0}}(\sigma(\omega))\right)}{\left(\varphi_{i}^{\gamma_{0}}\right)^{\prime}\left(\pi_{\gamma_{0}}(\sigma(\omega))\right)\left(\varphi_{i}^{\gamma_{0}}\right)^{\prime}\left(\pi_{\gamma_{0}}(\sigma(\tau))\right)}\right| \\
\leq & K \frac{\left|\left(\varphi_{i}^{\gamma_{0}}\right)^{\prime}\left(\beta_{\gamma_{0}, \tau}\right)\right|\left|\left(\varphi_{i}^{\gamma}\right)^{\prime}\left(\beta_{\gamma, \omega}\right)-\left(\varphi_{i}^{\gamma}\right)^{\prime}\left(\beta_{\gamma, \tau}\right)\right|}{\left|\left(\varphi_{i}^{\gamma_{0}}\right)^{\prime}\left(\beta_{\gamma_{0}, \tau}\right)\right|^{2}} \\
& \quad K \frac{\left|\left(\varphi_{i}^{\gamma}\right)^{\prime}\left(\beta_{\gamma, \tau}\right)\right|\left|\left(\varphi_{i}^{\gamma_{0}}\right)^{\prime}\left(\beta_{\gamma_{0}, \tau}\right)-\left(\varphi_{i}^{\gamma_{0}}\right)^{\prime}\left(\beta_{\gamma_{0}, \omega}\right)\right|}{\left|\left(\varphi_{i}^{\gamma_{0}}\right)^{\prime}\left(\beta_{\gamma_{0}, \tau}\right)\right|^{2}} .
\end{aligned}
$$


Let $r:=\operatorname{dist}\left(\partial V_{0}, X\right)$, where $V_{0}$ denotes the neighborhood of $X$ from Definition 3.2. Let $\left\{B\left(z_{j}, r / 2\right)\right\}_{j=1}^{J}$ be a finite covering of $X$ where $z_{j} \in X$ for each $j$. For each $1 \leq j \leq J$, $i \in I$ and $\gamma \in D_{d}\left(\gamma_{0}, R\right)$, we define $g_{j, i, \gamma}: B\left(z_{j}, r\right) \rightarrow \mathbb{C}$ as $g_{j, i, \gamma}(z)=\frac{\varphi_{i}^{\gamma}(z)}{\left(\varphi_{i}^{\gamma}\right)^{\prime}\left(z_{j}\right)}$. Then $g_{j, i, \gamma}$ is a univalent function on $B\left(z_{j}, r\right)$ such that $g_{j, i, \gamma}^{\prime}\left(z_{j}\right)=1$. By Koebe's Distortion Theorem, there exists a constant $M_{0} \geq 1$ such that for all $1 \leq j \leq J, i \in I$ and $\gamma \in D_{d}\left(\gamma_{0}, R\right)$, we have $M_{0}^{-1} \leq\left|g_{j, i, \gamma}^{\prime}\right| \leq M_{0}$ on $B\left(z_{j}, r / 2\right)$. Thus, there exists a constant $M_{1} \geq 1$ such that for all $i \in I, \gamma \in D_{d}\left(\gamma_{0}, R\right)$, and $a, b \in X$,

$$
M_{1}^{-1} \leq\left|\frac{\left(\varphi_{i}^{\gamma}\right)^{\prime}(b)}{\left(\varphi_{i}^{\gamma}\right)^{\prime}(a)}\right| \leq M_{1} .
$$

Combining this with Theorem 4.1.2 in [14], we obtain that there exists a constant $M_{2}>0$ such that for all $i \in I, \gamma \in D_{d}\left(\gamma_{0}, R\right)$ and $\omega, \tau \in I^{\infty}$ such that $\omega_{1}=\tau_{1}=i$,

$$
\begin{aligned}
\left|\left(\varphi_{i}^{\gamma}\right)^{\prime}\left(\beta_{\gamma, \omega}\right)-\left(\varphi_{i}^{\gamma}\right)^{\prime}\left(\beta_{\gamma, \tau}\right)\right| & \leq M_{2}\left|\left(\varphi_{i}^{\gamma}\right)^{\prime}\left(\beta_{\gamma, \tau}\right)\right|\left|\beta_{\gamma, \omega}-\beta_{\gamma, \tau}\right| \\
& \leq M_{2}\left|\left(\varphi_{i}^{\gamma}\right)^{\prime}\left(\beta_{\gamma, \tau}\right)\right| e^{-\alpha|\omega \wedge \tau|} \operatorname{diam}(X) .
\end{aligned}
$$

From (4.5), (4.6) and the assumption $\left|\kappa_{\omega}(\gamma)\right| \leq B$, it follows that there exists a constant $M_{3}>0$ such that for all $\omega, \tau \in I^{\infty}$ with $\omega_{1}=\tau_{1}=i$ and for all $\gamma \in D_{d}\left(\gamma_{0}, R\right)$,

$$
\left|\kappa_{\omega}(\gamma)-\kappa_{\tau}(\gamma)\right| \leq M_{3} e^{-\alpha|\omega \wedge \tau|}
$$

Applying Cauchy's formula to the well-defined branch $\log : B(1,1 / 2) \rightarrow \mathbb{C}$ with $\log 1=0$, which is bounded, we obtain that there exists a constant $M_{4}>0$ such that

$$
\left|\log \kappa_{\omega}(\gamma)-\log \kappa_{\tau}(\gamma)\right| \leq M_{4} e^{-\alpha|\omega \wedge \tau|}
$$

for all $\omega, \tau \in I^{\infty}$ with $\omega_{1}=\tau_{1}=i$ and for all $\gamma \in D_{d}\left(\gamma_{0}, R\right)$. The claim holds with $C_{0}=$ $\max \left\{2 M, M_{4}\right\}$.

Since by Lemma 4.11 the operator $\mathcal{R}_{d}^{\gamma_{0}}: \operatorname{Hol}\left(D_{b}^{d}\left(\gamma_{0}, R\right)\right) \rightarrow \operatorname{Hol}\left(D_{b}^{2 d}\left(I\left(\gamma_{0}\right), R / 4\right)\right)$ is linear and bounded with norm bounded above by $4^{d}$, we get that for all $\tilde{\gamma} \in D_{2 d}\left(I\left(\gamma_{0}\right), R / 4\right)$,

$$
\left|\mathcal{R}_{d}^{\gamma_{0}}\left(\log \kappa_{\omega}\right)(\tilde{\gamma})-\mathcal{R}_{d}^{\gamma_{0}}\left(\log \kappa_{\tau}\right)(\tilde{\gamma})\right|=\left|\mathcal{R}_{d}^{\gamma_{0}}\left(\log \kappa_{\omega}-\log \kappa_{\tau}\right)(\tilde{\gamma})\right| \leq 4^{d} C_{0} e^{-\alpha|\omega \wedge \tau|} .
$$

Hence, $\mathcal{R}_{d}^{\gamma_{0}}(\log \kappa)(\tilde{\gamma}) \in \mathcal{H}_{\alpha}$ and $v_{\alpha}\left(\mathcal{R}_{d}^{\gamma_{0}}(\log \kappa)(\tilde{\gamma})\right) \leq 4^{d} C_{0}$ for all $\tilde{\gamma} \in D_{2 d}\left(I\left(\gamma_{0}\right), R / 4\right)$. Combining this with (4.4) we deduce that $\left\|\mathcal{R}_{d}^{\gamma_{0}}(\log \kappa)(\tilde{\gamma})\right\|_{\alpha} \leq 4^{d}\left(M+C_{0}\right)$. Thus Condition (a) of Lemma 4.16 is satisfied. The proof is complete.

We are finally in a position to prove our theorem.

Proof of Theorem 4.10. Let $\gamma_{0}, R_{0}, s, \alpha$, and $R$ be as in Lemma 4.23. Let further $\tilde{\Gamma}=\mathbb{C}^{2 d}, G=D_{2 d}\left(I\left(\gamma_{0}\right), R / 4\right), \tilde{\gamma}_{0}=I\left(\gamma_{0}\right), \Phi^{\tilde{\gamma}_{0}}=\Phi^{\gamma_{0}}$, and $g_{\tilde{\gamma}}(\omega)=t \mathcal{R}_{d}^{\gamma_{0}}\left(\log \kappa_{\omega}\right)(\tilde{\gamma})$, as in the proof of Lemma 4.23. Recall that according to that lemma, the operator $\mathcal{L}_{\tilde{\gamma}}: \mathcal{H}_{\alpha} \rightarrow$ $\mathcal{H}_{\alpha}$ generated by the potential $u_{\tilde{\gamma}}(\omega):=t \mathcal{R}_{d}^{\gamma_{0}}\left(\log \kappa_{\omega}\right)(\tilde{\gamma})+t \log \left|\left(\varphi_{\omega_{1}}^{\gamma_{0}}\right)^{\prime}\left(\pi_{\gamma_{0}}(\sigma(\omega))\right)\right|$ depends 
holomorphically on $\tilde{\gamma} \in D_{2 d}\left(I\left(\gamma_{0}\right), R / 4\right)$. Note that, because of the definition of the function $\kappa$, we have

$$
\exp \left(t \mathcal{R}_{d}^{\gamma_{0}}\left(\log \kappa_{\omega}\right)(\gamma)+t \log \left|\left(\varphi_{\omega_{1}}^{\gamma_{0}}\right)^{\prime}\left(\pi_{\gamma_{0}}(\sigma(\omega))\right)\right|\right)=\left|\left(\varphi_{\omega_{1}}^{\gamma}\right)^{\prime}\left(\pi_{\gamma}(\sigma(\omega))\right)\right|^{t}
$$

for all $\gamma \in D_{d}\left(\gamma_{0}, R / 4\right)$, where $D_{d}\left(\gamma_{0}, R / 4\right)$ is identified with $D_{2 d}\left(I\left(\gamma_{0}\right), R / 4\right) \cap \mathbb{R}^{2 d}$ as $\mathbb{C}^{d}$ is embedded in $\mathbb{C}^{2 d}$ by the formula $\left(x_{1}+i y_{1}, x_{2}+i y_{2}, \ldots, x_{d}+i y_{d}\right) \mapsto\left(x_{1}, y_{1}, x_{2}, y_{2}, \ldots, x_{d}, y_{d}\right)$. Thus, in view of (4.7) and Theorem 2.4.6 and Corollary 2.7.5 from [14], $e^{P\left(\gamma_{0}, t\right)}$ is a simple isolated eigenvalue of the operator $\mathcal{L}_{I\left(\gamma_{0}\right)}: \mathcal{H}_{\alpha} \rightarrow \mathcal{H}_{\alpha}$. Hence, in view of Lemmas 4.16 and 4.23, which ensure the analyticity of the function $\tilde{\gamma} \mapsto \mathcal{L}_{\tilde{\gamma}}$ from $D_{2 d}\left(I\left(\gamma_{0}\right), R / 4\right)$ to $L\left(\mathcal{H}_{\alpha}\right)$, KatoRellich Perturbation Theorem (see [10], Theorem 3.16 on page 212 and pages 368-369) yields $r_{1} \in(0, R / 4]$ and a holomorphic function $\xi: D_{2 d}\left(I\left(\gamma_{0}\right), r_{1}\right) \rightarrow \mathbb{C}$ such that $\xi\left(I\left(\gamma_{0}\right)\right)=e^{P\left(\gamma_{0}, t\right)}$ and such that for each $\tilde{\gamma} \in D_{2 d}\left(I\left(\gamma_{0}\right), r_{1}\right)$, it turns out that $\xi(\tilde{\gamma})$ is a simple isolated eigenvalue of $\mathcal{L}_{\tilde{\gamma}}: \mathcal{H}_{\alpha} \rightarrow \mathcal{H}_{\alpha}$ with the remainder of the spectrum uniformly separated from $\xi(\tilde{\gamma})$. In particular, there exists $r_{2} \in\left(0, r_{1}\right]$ and $\eta>0$ such that

$$
\sigma\left(\mathcal{L}_{\tilde{\gamma}}\right) \cap B\left(e^{P\left(\gamma_{0}, t\right)}, \eta\right)=\{\xi(\tilde{\gamma})\}
$$

for all $\tilde{\gamma} \in D_{2 d}\left(I\left(\gamma_{0}\right), r_{2}\right)$. Since $e^{P(\gamma, t)}$ is the spectral radius of the operator $\mathcal{L}_{\gamma}$ for all $\gamma \in D_{d}\left(\gamma_{0}, r_{2}\right)$ (see Theorem 2.4.6 and Corollary 2.7.5 in [14]), in view of the semi-continuity of the spectral set function (see Theorem 10.20 in [19]), taking $r_{2}>0$ small enough, we also have that the spectral radius of $\mathcal{L}_{\gamma}$ is in $\left[0, e^{P\left(\gamma_{0}, t\right)}+\eta\right)$, and along with (4.8), this implies that $e^{P(\gamma, t)}=\xi(\gamma)$ for all $\gamma \in D_{d}\left(\gamma_{0}, r_{2}\right)$. Consequently, the function $\gamma \mapsto P(\gamma, t), \gamma \in D_{d}\left(\gamma_{0}, r_{2}\right)$, is real-analytic. The proof of Theorem 4.10 is complete.

Remark 4.24. Condition (1) in Theorem 4.10 is a local version of condition (c) in [16].

Those analytic families that depend continuously on the parameter $\gamma$ when $\operatorname{HIFS}(X)$ is equipped with the $\lambda$-topology satisfy condition (1).

Lemma 4.25. Let $\left\{\Phi^{\gamma}\right\}_{\gamma \in \Gamma}$ be an analytic family such that $\gamma \mapsto \Phi^{\gamma} \in \operatorname{HIFS}(X)$ is continuous with respect to the $\lambda$-topology. Then condition (1) in Theorem 4.10 holds.

Proof. This lemma is a consequence of Lemma 4.21 and the observation following Corollary 6.2 in [16].

Remark 4.7, Theorems 4.9 and 4.10, and Lemma 4.25 justify the assumption of continuous dependence on $\gamma$ of the families studied in the sequel.

\section{A Classification Theorem}

We now give a complete classification of analytic families which are continuous with respect to the $\lambda$-topology.

Definition 5.1. Let $\left\{\Phi^{\gamma}\right\}_{\gamma \in \Gamma}$ be an analytic family in $\operatorname{HIFS}(X)$. We define the following subsets of parameters: 
- $\mathrm{R}_{\Gamma}(X):=\left\{\gamma \in \Gamma: \Phi^{\gamma} \in \mathrm{R}(X)\right\}$

- $\operatorname{SR}_{\Gamma}(X):=\left\{\gamma \in \Gamma: \Phi^{\gamma} \in \operatorname{SR}(X)\right\}$;

- $\operatorname{CFR}_{\Gamma}(X):=\left\{\gamma \in \Gamma: \Phi^{\gamma} \in \operatorname{CFR}(X)\right\}$;

- $\operatorname{FSR}_{\Gamma}(X):=\left\{\gamma \in \Gamma: \Phi^{\gamma} \in \mathrm{SR}(X) \backslash \mathrm{CFR}(X)\right\}$;

- $\mathrm{CR}_{\Gamma}(X):=\left\{\gamma \in \Gamma: \Phi^{\gamma} \in \mathrm{CR}(X)\right\}$;

- $\operatorname{IR}_{\Gamma}(X):=\left\{\gamma \in \Gamma: \Phi^{\gamma} \in \operatorname{IR}(X)\right\}$;

- $\operatorname{RAH}(\Gamma):=\left\{\gamma_{0} \in \Gamma: \gamma \mapsto h_{\Phi^{\gamma}}\right.$ is real-analytic in a neighborhood of $\left.\gamma_{0}\right\}$; and

- $\operatorname{NPHH}(\Gamma):=\left\{\gamma_{0} \in \Gamma: \gamma \mapsto h_{\Phi^{\gamma}}\right.$ is not pluriharmonic in any neighborhood of $\left.\gamma_{0}\right\}$.

Theorem 5.2. Let $\left\{\Phi^{\gamma}\right\}_{\gamma \in \Gamma}$ be an analytic family in $\operatorname{HIFS}(X)$ such that $\gamma \mapsto \Phi^{\gamma} \in \operatorname{CIFS}(X)$ is continuous with respect to the $\lambda$-topology. Let $\theta$ be the constant such that $\theta_{\Phi}=\theta$ on $\Gamma$ (this constant exists according to Lemma 5.4 in [16]). Then exactly one of the following statements holds.

(I) $\Gamma=\operatorname{CFR}_{\Gamma}(X)=\operatorname{RAH}(\Gamma)$, and the function $\gamma \mapsto h_{\Phi^{\gamma}}, \gamma \in \Gamma$, is identically equal to a constant larger than $\theta$.

(II) $\Gamma=\operatorname{CFR}_{\Gamma}(X)=\operatorname{RAH}(\Gamma)=\operatorname{NPHH}(\Gamma)$.

(III) $\Gamma=\operatorname{FSR}_{\Gamma}(X)=\operatorname{RAH}(\Gamma)$, and the function $\gamma \mapsto h_{\Phi^{\gamma}}, \gamma \in \Gamma$, is identically equal to a constant larger than $\theta$.

(IV) $\Gamma=\operatorname{FSR}_{\Gamma}(X)=\operatorname{RAH}(\Gamma)=\operatorname{NPHH}(\Gamma)$.

(V) $\Gamma=\operatorname{FSR}_{\Gamma}(X) \cup \mathrm{CR}_{\Gamma}(X)=\operatorname{NPHH}(\Gamma)$, with $\operatorname{FSR}_{\Gamma}(X) \neq \emptyset$ and $\mathrm{CR}_{\Gamma}(X) \neq \emptyset$.

Furthermore, $\mathrm{CR}_{\Gamma}(X)=\partial\left(\operatorname{FSR}_{\Gamma}(X)\right)$.

Also, $\operatorname{FSR}_{\Gamma}(X) \subset \mathrm{RAH}(\Gamma)$.

(VI) $\Gamma=\mathrm{CR}_{\Gamma}(X)=\operatorname{RAH}(\Gamma)$, and the function $\gamma \mapsto h_{\Phi^{\gamma}}, \gamma \in \Gamma$, is identically equal to the constant $\theta$.

(VII) $\operatorname{FSR}_{\Gamma}(X) \neq \emptyset, \mathrm{CR}_{\Gamma}(X) \neq \emptyset$ and $\operatorname{IR}_{\Gamma}(X) \neq \emptyset$, while $\mathrm{CFR}_{\Gamma}(X)=\emptyset$.

Moreover, $\emptyset \neq \partial\left(\operatorname{IR}_{\Gamma}(X)\right) \subset \mathrm{CR}_{\Gamma}(X)=\partial\left(\operatorname{FSR}_{\Gamma}(X)\right)$.

Moreover, $\operatorname{FSR}_{\Gamma}(X) \cup \operatorname{IR}_{\Gamma}(X) \subset \operatorname{RAH}(\Gamma)$.

Moreover, $\emptyset \neq \partial\left(\operatorname{IR}_{\Gamma}(X)\right) \subset \Gamma \backslash \operatorname{RAH}(\Gamma)$.

Furthermore, $\mathrm{NPHH}(\Gamma)=\operatorname{FSR}_{\Gamma}(X) \cup \mathrm{CR}_{\Gamma}(X)$.

(VIII) $\Gamma=\operatorname{IR}_{\Gamma}(X)=\operatorname{RAH}(\Gamma)$, and the function $\gamma \mapsto h_{\Phi^{\gamma}}, \gamma \in \Gamma$, is identically equal to the constant $\theta$.

Proof. Case (1): Suppose that $\operatorname{CFR}_{\Gamma}(X) \neq \emptyset$. Since $\operatorname{CFR}_{\Gamma}(X)$ is clopen by Lemma 5.9 in [16] and $\Gamma$ is connected, we deduce that $\Gamma=\operatorname{CFR}_{\Gamma}(X)$. Now, if the function $\gamma \mapsto h_{\Phi^{\gamma}}$, $\gamma \in \Gamma$, is not constant, then combining Theorem 6.1 and Corollary 6.4 in [16] we obtain $\Gamma=\operatorname{RAH}(\Gamma)=\operatorname{NPHH}(\Gamma)$. This corresponds to statement (II).

On the other hand, if the function $\gamma \mapsto h_{\Phi^{\gamma}}, \gamma \in \Gamma$, is identically equal to a constant $\tau$, then by Theorem 2.4 in [16] we have $\tau>\theta$. This is statement (I).

Case (2): Suppose next that $\Gamma=\operatorname{FSR}_{\Gamma}(X)$. If $\gamma \mapsto h_{\Phi^{\gamma}}, \gamma \in \Gamma$, is not constant, then combining Theorem 6.1 and Corollary 6.4 in [16] we get $\Gamma=\operatorname{RAH}(\Gamma)=\operatorname{NPHH}(\Gamma)$. This is statement (IV). 
If, however, the function $\gamma \mapsto h_{\Phi^{\gamma}}, \gamma \in \Gamma$, is identically equal to a constant $\tau$, then by Theorem 2.4 in [16] we have $\tau>\theta$. This is statement (III).

Case (3): Suppose that $\Gamma=\operatorname{FSR}_{\Gamma}(X) \cup \mathrm{CR}_{\Gamma}(X)$, that $\operatorname{FSR}_{\Gamma}(X) \neq \emptyset$, and $\mathrm{CR}_{\Gamma}(X) \neq \emptyset$. Then, by Lemma 5.9 in [16] we know that $\operatorname{FSR}_{\Gamma}(X)$ is a proper non-empty open subset of $\Gamma$. Moreover, by Theorem 6.1 in [16] we have $\operatorname{FSR}_{\Gamma}(X) \subset \operatorname{RAH}(\Gamma)$. Now, if there exists a parameter $\gamma_{0} \in \operatorname{FSR}_{\Gamma}(X)$ such that $\gamma \mapsto h_{\Phi^{\gamma}}$ is pluriharmonic around $\gamma_{0}$, then by Corollary 6.4 and Theorem 2.4 in [16], we deduce that the function $\gamma \mapsto h_{\Phi \gamma}$ is identically equal to a constant $\tau>\theta$ in the connected component $U_{0}$ of $\operatorname{FSR}_{\Gamma}(X)$ containing $\gamma_{0}$. Since $\mathrm{CR}_{\Gamma}(X) \neq \emptyset$ by assumption and since $\Gamma$ is connected, the boundary $\partial U_{0}$ of $U_{0}$ in $\Gamma$ is not empty. Moreover, $\partial U_{0} \subset \mathrm{CR}_{\Gamma}(X)$. Then, according to Theorem 5.10 in [16], the function $\gamma \mapsto h_{\Phi^{\gamma}}$ is continuous and therefore $h_{\Phi^{\gamma}}=\tau$ for every $\gamma \in \partial U_{0}$. However, using Theorem 2.3 in [16], we know that $h_{\Phi \gamma}=\theta$ for every $\gamma \in \mathrm{CR}_{\Gamma}(X)$. This is a contradiction. Consequently, $\overline{\mathrm{FSR}_{\Gamma}(X)} \subset$ $\operatorname{NPHH}(\Gamma)$. Furthermore, Theorem 4.10 above and Theorems 2.3 and 2.4 in [16] imply that $\mathrm{CR}_{\Gamma}(X)=\partial\left(\mathrm{FSR}_{\Gamma}(X)\right)$. Thus, we obtain $\Gamma=\overline{\operatorname{FSR}_{\Gamma}(X)}=\operatorname{NPHH}(\Gamma)$. This means that, in this case, statement $(\mathrm{V})$ holds.

Case (4): Suppose next that $\Gamma=\mathrm{CR}_{\Gamma}(X)$. Then, by Theorem 2.3 in [16], statement (VI) holds.

Case (5): Suppose now that $\Gamma=\operatorname{IR}_{\Gamma}(X)$. Then, by Theorem 2.3 in [16], statement (VIII) holds.

Case (6): Suppose next that $\operatorname{CR}_{\Gamma}(X) \neq \emptyset$ and $\operatorname{IR}_{\Gamma}(X) \neq \emptyset$. Then $\gamma \mapsto P(\gamma, \theta)$ is not constant on $\Gamma$. Theorems 4.10 and 4.9 imply that the function $\gamma \mapsto P(\gamma, \theta)$ is a non-constant real-analytic function that satisfies the Maximum Principle. It follows that for any parameter $\gamma_{0} \in \mathrm{CR}_{\Gamma}(X)$, there exists a parameter $\gamma$, arbitrarily close to $\gamma_{0}$, such that $P(\gamma, \theta)>0$. Notice that $P(\gamma, \theta)>0$ implies that $\gamma \in \mathrm{SR}_{\Gamma}(X)$. Hence, $\mathrm{CR}_{\Gamma}(X) \subset \partial\left(\operatorname{FSR}_{\Gamma}(X)\right)$. On the other hand, the continuity of the function $\gamma \mapsto P(\gamma, \theta)$ implies that $\partial\left(\operatorname{FSR}_{\Gamma}(X)\right) \subset \mathrm{CR}_{\Gamma}(X)$. Thus, $\partial\left(\mathrm{FSR}_{\Gamma}(X)\right)=\mathrm{CR}_{\Gamma}(X)$. The continuity of the function $\gamma \mapsto P(\gamma, \theta)$ also implies that $\partial\left(\operatorname{IR}_{\Gamma}(X)\right) \subset \mathrm{CR}_{\Gamma}(X)$, while the connectedness of $\Gamma$ ensures that $\partial\left(\operatorname{IR}_{\Gamma}(X)\right) \neq \emptyset$.

Moreover, using Theorems 5.10 and 6.1 in [16], we deduce that $\operatorname{FSR}_{\Gamma}(X) \cup \operatorname{IR}_{\Gamma}(X) \subset$ $\operatorname{RAH}(\Gamma)$.

In order to show that $\partial\left(\operatorname{IR}_{\Gamma}(X)\right) \subset \Gamma \backslash \operatorname{RAH}(\Gamma)$, let $\gamma_{0} \in \partial\left(\operatorname{IR}_{\Gamma}(X)\right)$. Then, $\gamma_{0} \in \mathrm{CR}_{\Gamma}(X)=$ $\partial\left(\operatorname{FSR}_{\Gamma}(X)\right)$. If $\gamma_{0} \in \operatorname{RAH}(\Gamma)$, then the function $\gamma \mapsto h_{\Phi^{\gamma}}$ is identically equal to the constant $\theta$ in a neighborhood of $\gamma_{0}$. However, this contradicts Theorem 2.4 in [16]. Hence, we obtain that $\partial\left(\operatorname{IR}_{\Gamma}(X)\right) \subset \Gamma \backslash \operatorname{RAH}(\Gamma)$.

By the same argument as in case (3), we obtain $\operatorname{NPHH}(\Gamma)=\mathrm{FSR}_{\Gamma}(X) \cup \mathrm{CR}_{\Gamma}(X)$.

Thus, in this case, statement (VII) holds.

Case (7): Finally, suppose that $\operatorname{FSR}_{\Gamma}(X) \neq \emptyset$ and $\operatorname{IR}_{\Gamma}(X) \neq \emptyset$. Then, by Theorem 5.7 in [16], we have $\mathrm{CR}_{\Gamma}(X) \neq \emptyset$. Therefore, by case (6), statement (VII) holds.

We are done.

This classification theorem further highlights some differences between analytic families and the entire space $\operatorname{CIFS}(X)$. Whereas we know that $\partial(\operatorname{IR}(X))=\operatorname{CR}(X)$ while $\partial(\operatorname{SR}(X)) \subset$ 
$\mathrm{CR}(X)$ in $\operatorname{CIFS}(X)$, there exist analytic families of types (V) and (VII) (see Example 5.4, Theorem 6.5, and Proposition 6.6).

Corollary 5.3. Under the assumptions of Theorem 5.2, the set $\Gamma \backslash \mathrm{RAH}(\Gamma)$ is included in a proper real-analytic subvariety of $\Gamma$. In particular, $\mathrm{RAH}(\Gamma)$ is open and dense in $\Gamma$.

Proof. If the family $\left\{\Phi^{\gamma}\right\}_{\gamma \in \Gamma}$ is of type (V) or (VII), then $\Gamma \backslash \mathrm{RAH}(\Gamma)$ is included in the set $\mathrm{CR}_{\Gamma}(X)=\{\gamma \in \Gamma \mid P(\gamma, \theta)=0\}$, which is a proper real-analytic subset of $\Gamma$ due to Theorem 4.10 .

Note that every type of family described in Theorem 5.2 exists (see Proposition 6.6). It is indeed possible to construct families of every type and in the following example we present a family of type $(\mathrm{V})$ in $\operatorname{HIFS}(\overline{\mathbb{D}})$, where $\mathbb{D}=B(0,1) \subset \mathbb{C}$ is the open unit disk and $\overline{\mathbb{D}}=$ $\overline{B(0,1)} \subset \mathbb{C}$ is the closed unit disk.

Example 5.4. Let $U$ be a non-empty open subset of $\mathbb{D}$ and let $z_{1}, z_{2}, z_{3} \in U$ be mutually distinct points. Let $\left\{\alpha_{i}(z):=a_{i}\left(z-z_{i}\right)+z_{i}\right\}_{i=1}^{3}$ be such that $0<a_{1}=a_{2}=a<1 / 2$, $0<a_{3}<1, \bigcup_{i=1}^{3} \alpha_{i}(\bar{D}) \subset U$, and $\alpha_{i}(\bar{D}) \cap \alpha_{j}(\bar{D})=\emptyset$ whenever $i \neq j$. Let $\left\{c_{i}\right\}_{i \geq 3}$ be $a$ sequence of positive numbers such that

$$
\sum_{i=3}^{\infty} c_{i}^{t}= \begin{cases}\infty & \text { if } t<1 \\ \frac{1-2 a}{a_{3}} & \text { if } t=1 \\ <\infty & \text { if } t>1\end{cases}
$$

Let $\Gamma=B(0, r) \subset \mathbb{C}$ be a small disk around 0 and $X=\overline{\mathbb{D}}$. For each $\gamma \in \Gamma$, let

$$
\Phi^{\gamma}:=\left\{\begin{array}{l}
\varphi_{1}^{\gamma}(z):=4 a\left(\frac{1}{2}+\gamma\right)^{2}\left(z-z_{1}\right)+z_{1} \\
\varphi_{2}^{\gamma}(z):=4 a\left(\frac{1}{2}-\gamma\right)^{2}\left(z-z_{2}\right)+z_{2} \\
\varphi_{i}^{\gamma}(z):=a_{3}\left(\psi_{i}(z)-z_{3}\right)+z_{3}, \quad i \geq 3
\end{array}\right.
$$

where $\left\{\psi_{i}\right\}_{i \geq 3}$ is a family of similitudes such that $\psi_{i}(\mathbb{D}) \subset \mathbb{D}, \psi_{i}(\mathbb{D}) \cap \psi_{j}(\mathbb{D})=\emptyset$ whenever $i \neq j$, and $\psi_{i}^{\prime}(z) \equiv c_{i}$. (Note that by Lemma 3.2(i) in [17], such $\left\{c_{i}\right\}_{i \geq 3}$ and $\left\{\psi_{i}\right\}_{i \geq 3}$ exist.) We claim that the family $\left\{\Phi^{\gamma}\right\}_{\gamma \in \Gamma}$ is of type $(V)$ and $\varphi_{i}^{\gamma}(\bar{D}) \subset U$ for all $\gamma \in \Gamma$ and all $i \in I$.

Indeed, if we take $r$ so small, $\left\{\Phi^{\gamma}\right\}_{\gamma \in \Gamma}$ is an analytic family in $\operatorname{HIFS}(\overline{\mathbb{D}})$ and $\varphi_{i}^{\gamma}(\overline{\mathbb{D}}) \subset U$ for all $\gamma \in \Gamma$ and all $i \in I$. Moreover, $\gamma \mapsto \Phi^{\gamma}$ is continuous with respect to the $\lambda$-topology.

Furthermore,

$$
P(\gamma, t)=\log \left((4 a)^{t}\left|\frac{1}{2}+\gamma\right|^{2 t}+(4 a)^{t}\left|\frac{1}{2}-\gamma\right|^{2 t}+a_{3}^{t} \sum_{i=3}^{\infty} c_{i}^{t}\right)
$$


Therefore $\theta_{\Phi \gamma}=1$ for every $\gamma$ and

$$
\begin{aligned}
P\left(\gamma, \theta_{\Phi^{\gamma}}\right)=P(\gamma, 1) & =\log \left(4 a\left|\frac{1}{2}+\gamma\right|^{2}+4 a\left|\frac{1}{2}-\gamma\right|^{2}+a_{3} \sum_{i=3}^{\infty} c_{i}\right) \\
& =\log \left(4 a\left(\frac{1}{2}+2|\gamma|^{2}\right)+a_{3} \cdot \frac{1-2 a}{a_{3}}\right) \\
& =\log \left(1+8 a|\gamma|^{2}\right) .
\end{aligned}
$$

This latter function of $\gamma$ takes its minimum value 0 at $\gamma=0$ and is positive for each small $\gamma \neq 0$. Thus, $\left\{\Phi^{\gamma}\right\}_{\gamma \in \Gamma}$ is of type $(\mathrm{V})$.

\section{Consequences of the Classification Theorem}

Propositions 5.13-5.16 in [17] assert that $\partial(\operatorname{SR}(X))=\partial(\operatorname{FSR}(X)) \subset \mathrm{CR}(X)$, despite that $\mathrm{CR}(X) \neq \partial(\operatorname{SR}(X))$. Moreover, Proposition 5.17 of that same paper provides a condition under which a critically regular SIFS is in the boundary of the subset of strongly regular CIFSs. Using our classification theorem, we partially generalize this result. In this section, let $X$ be a non-empty compact connected subset of $\mathbb{C}$ such that $X=\overline{\operatorname{Int}(X)}$ and the condition (iii) in section 2 is satisfied.

Theorem 6.1. Suppose that $X \subset \mathbb{C}$ is star shaped with $x_{0} \in \operatorname{Int}(X)$ for center. Let $\Phi=$ $\left\{\varphi_{i}\right\} \in \mathrm{CR}(X) \cap \operatorname{HIFS}(X)$ be a system such that there exists a finite set $F \subset \mathbb{N}$ for which

$$
\bigcup_{i \in F} \varphi_{i}(X) \subset \operatorname{Int}(X) \backslash \overline{\bigcup_{j \notin F} \varphi_{j}(X)} \neq \emptyset .
$$

Then $\Phi \in \partial(\operatorname{SR}(X))=\partial(\operatorname{FSR}(X))$. More precisely, there exists an open, connected neighborhood $\Gamma$ of $\{s \in \mathbb{R}: 0<s \leq 1\}$ in $\mathbb{C}$ such that, setting

$$
\Phi^{\gamma}=\left\{\begin{array}{ll}
\varphi_{i}, & i \notin F, \\
\varphi_{i} \circ \psi_{\gamma}, & i \in F
\end{array}\right\}
$$

for each $\gamma \in \Gamma$, where $\psi_{\gamma}(x)=\gamma\left(x-x_{0}\right)+x_{0}$, the analytic family $\left\{\Phi^{\gamma}\right\}_{\gamma \in \Gamma}$ in $\operatorname{HIFS}(X)$ satisfies all of the following:

(1) $\Phi^{1}=\Phi$;

(2) $\gamma \in \Gamma \mapsto \Phi^{\gamma} \in \operatorname{HIFS}(X)$ is continuous with respect to the $\lambda$-topology;

(3) $\left\{\Phi^{\gamma}\right\}_{\gamma \in \Gamma}$ is of type (VII);

(4) $1 \in \mathrm{CR}_{\Gamma}(X)=\partial\left(\operatorname{FSR}_{\Gamma}(X)\right)$;

(5) $\{s \in \mathbb{R}: 0<s<1\} \cap \operatorname{IR}_{\Gamma}(X) \neq \emptyset$ and $\{s \in \mathbb{R}: 0<s \leq 1\} \cap \partial\left(\operatorname{IR}_{\Gamma}(X)\right) \neq \emptyset$; and

(6) for any $\gamma_{0} \in \partial\left(\operatorname{IR}_{\Gamma}(X)\right)$, we have $\gamma_{0} \in \partial\left(\operatorname{IR}_{\Gamma}(X)\right) \subset \mathrm{CR}_{\Gamma}(X)=\partial\left(\operatorname{FSR}_{\Gamma}(X)\right)$ and $\gamma \mapsto h_{\Phi \gamma}$ is not real-analytic in any neighborhood of $\gamma_{0}$ in $\Gamma$.

Proof. Using the local bounded distortion property (see Corollary 4.1 .4 in [14]), the fact that all generators $\varphi_{i}, i \in \mathbb{N}$, are contractions with a common ratio, and the Mean Value Inequality, we can construct a decreasing family $\mathcal{F}$ of open, connected neighborhoods of $X$ whose intersection is $X$ and with respect to each of which $\Phi$ is in $\operatorname{HIFS}(X)$. (In fact, 
$X_{\varepsilon}:=B(X, \varepsilon)$ is such a neighborhood for every $\varepsilon>0$ small enough). This family $\mathcal{F}$ therefore has a member $U \supset X$ such that

$$
\bigcup_{i \in F} \varphi_{i}(\bar{U}) \subset \operatorname{Int}(X) \backslash \overline{\bigcup_{j \notin F} \varphi_{j}(X)} \neq \emptyset .
$$

Choose $\delta>0$ such that $\overline{X_{\delta}} \subset U$. Thereafter choose an open neighborhood $\Gamma$ of $\{s \in \mathbb{R}: 0<$ $s \leq 1\}$ in $\mathbb{C}$ such that

$$
\bigcup_{\gamma \in \Gamma} \psi_{\gamma}\left(\overline{X_{\delta}}\right) \subset U
$$

Finally, take for $V$ any neighborhood in the family $\mathcal{F}$ such that $V \subset X_{\delta}$. Then $\varphi_{i}(V) \subset V$ for all $i \in \mathbb{N}$ and

$$
\varphi_{k} \circ \psi_{\gamma}(V) \subset \varphi_{k} \circ \psi_{\gamma}\left(\overline{X_{\delta}}\right) \subset \varphi_{k}(U) \subset \operatorname{Int}(X) \backslash \overline{\cup_{j \notin F} \varphi_{j}(X)}
$$

for every $k \in F$ and every $\gamma \in \Gamma$. With this neighborhood $V$, we have that $\left\{\Phi^{\gamma}\right\}_{\gamma \in \Gamma}$ is an analytic family in $\operatorname{HIFS}(X)$. Moreover, $\gamma \mapsto \Phi^{\gamma} \in \operatorname{HIFS}(X)$ is clearly continuous with respect to the $\lambda$-topology. Furthermore, if $s>0$ is small enough, then by the argument given in the proof of Proposition 5.11 in [17], we obtain that $s \in \mathrm{IR}_{\Gamma}(X)$. Thus, Theorem 5.2 implies that the analytic family $\left\{\Phi^{\gamma}\right\}_{\gamma \in \Gamma}$ is of type (VII). In particular, $1 \in \mathrm{CR}_{\Gamma}(X)=\partial\left(\mathrm{FSR}_{\Gamma}(X)\right)$.

Now, let

$$
S:=\sup \left\{s \in \mathbb{R}: 0<s<1, s \in \operatorname{IR}_{\Gamma}(X)\right\} .
$$

Then $S \in \partial\left(\operatorname{IR}_{\Gamma}(X)\right)$. Thus, statement (5) holds.

Finally, by Theorem 5.2, statement (6) holds.

By a similar argument, we get the following, more general result.

Theorem 6.2. Let $X \subset \mathbb{C}$ and $\Phi=\left\{\varphi_{i}\right\} \in \mathrm{CR}(X) \cap \operatorname{HIFS}(X)$ be such that there exist a finite set $F \subset \mathbb{N}$ and a simply connected subdomain $W$ of $\operatorname{Int}(X)$ such that

$$
\bigcup_{i \in F} \varphi_{i}(X) \subset W \text { and } W \bigcap \overline{\bigcup_{j \notin F} \varphi_{j}(X)}=\emptyset
$$

Then $\Phi \in \partial(\operatorname{SR}(X))=\partial(\operatorname{FSR}(X))$. More precisely, there exists an analytic family $\left\{\Phi^{\gamma}\right\}_{\gamma \in \Gamma}$ in $\operatorname{HIFS}(X)$ with an open, connected neighborhood $\Gamma$ of $\{s \in \mathbb{R}: 0<s \leq 1\}$ in $\mathbb{C}$ such that

(1) $\Phi^{1}=\Phi$;

(2) $\gamma \in \Gamma \mapsto \Phi^{\gamma} \in \operatorname{HIFS}(X)$ is continuous with respect to the $\lambda$-topology;

(3) $\left\{\Phi^{\gamma}\right\}_{\gamma \in \Gamma}$ is of type (VII);

(4) $1 \in \partial\left(\operatorname{FSR}_{\Gamma}(X)\right)$;

(5) $\{s \in \mathbb{R}: 0<s<1\} \cap \operatorname{IR}_{\Gamma}(X) \neq \emptyset$ and $\{s \in \mathbb{R}: 0<s \leq 1\} \cap \partial\left(\operatorname{IR}_{\Gamma}(X)\right) \neq \emptyset$; and

(6) for any $\gamma_{0} \in \partial\left(\operatorname{IR}_{\Gamma}(X)\right)$, we have $\gamma_{0} \in \partial\left(\operatorname{IR}_{\Gamma}(X)\right) \subset \mathrm{CR}_{\Gamma}(X)=\partial\left(\operatorname{FSR}_{\Gamma}(X)\right)$ and $\gamma \mapsto h_{\Phi^{\gamma}}$ is not real-analytic in any neighborhood of $\gamma_{0}$ in $\Gamma$.

Proof. Let $A$ be a simply connected subdomain of $W$ such that $\cup_{i \in F} \varphi_{i}(X) \subset A \subset \bar{A} \subset W$. Let also $a \in A$. Taking an appropriate $A$, the Riemann Mapping Theorem implies that there exists an analytic family $\left\{\psi_{\gamma}\right\}_{\gamma \in \Gamma}$ of injective holomorphic maps from $A$ to $W$ with an open, 
connected neighborhood $\Gamma$ of $\{s \in \mathbb{R}: 0<s \leq 1\}$ in $\mathbb{C}$ such that $\psi_{1}(z) \equiv z$ and $\psi_{s}(z) \rightarrow a$ as $|s| \rightarrow 0$ uniformly on $A$.

For each $\gamma \in \Gamma$, let

$$
\Phi^{\gamma}=\left\{\begin{array}{ll}
\varphi_{i}, & i \notin F \\
\psi_{\gamma} \circ \varphi_{i}, & i \in F
\end{array}\right\}
$$

Then $\left\{\Phi^{\gamma}\right\}_{\gamma \in \Gamma}$ is an analytic family in $\operatorname{HIFS}(X)$. Note that a neighborhood common to all $\Phi^{\gamma}$ 's can be found as follows. As explained in the proof of Theorem 6.1, we can construct a decreasing family $\mathcal{F}$ of open, connected neighborhoods of $X$ whose intersection is $X$ and with respect to each of which $\Phi$ is in $\operatorname{HIFS}(X)$. This family therefore has a member $V \supset X$ such that $\cup_{i \in F} \varphi_{i}(V) \subset A$. Then $\varphi_{i}(V) \subset V$ for all $i \in \mathbb{N}$ and

$$
\psi_{\gamma} \circ \varphi_{i}(V) \subset \psi_{\gamma}(A) \subset W \subset \operatorname{Int}(X) \subset V
$$

for every $i \in F$. Moreover, $\gamma \in \Gamma \mapsto \Phi^{\gamma} \in \operatorname{HIFS}(X)$ is continuous with respect to the $\lambda$ topology. Furthermore, by the argument given in the proof of Proposition 5.11 in [17], every $\gamma \in \Gamma$ with small enough modulus is in $\operatorname{IR}_{\Gamma}(X)$. Hence, the family $\left\{\Phi^{\gamma}\right\}_{\gamma \in \Gamma}$ is of type (VII). In particular, $1 \in \mathrm{CR}_{\Gamma}(X)=\partial\left(\mathrm{FSR}_{\Gamma}(X)\right)$. The remaining statements follow from the same arguments as those given in the proof of Theorem 6.1.

We now give a better description of the set $\operatorname{FSR}(X) \cap \operatorname{HIFS}(X)$.

Proposition 6.3. Suppose that $X$ is star shaped with $x_{0} \in \operatorname{Int}(X)$ for center. Let $\Phi=\left\{\varphi_{i}\right\} \in$ $\operatorname{FSR}(X) \cap \operatorname{HIFS}(X)$. Then there exists an open, connected neighborhood $\Gamma$ of $\{s \in \mathbb{R}: 0<$ $s \leq 1\}$ in $\mathbb{C}$ such that, setting $\Phi^{\gamma}=\left\{\varphi_{i} \circ \psi_{\gamma}\right\}_{i \in I}$ for each $\gamma \in \Gamma$, where $\psi_{\gamma}(x)=\gamma\left(x-x_{0}\right)+x_{0}$, the analytic family $\left\{\Phi^{\gamma}\right\}_{\gamma \in \Gamma}$ in $\operatorname{HIFS}(X)$ satisfies

(1) $\Phi^{1}=\Phi$;

(2) $\Phi^{\gamma} \rightarrow \Phi$ as $\gamma \nearrow 1$ in $(0,1)$ with respect to the $\lambda$-topology;

(3) $\gamma \in \Gamma \mapsto \Phi^{\gamma} \in \operatorname{HIFS}(X)$ is continuous with respect to the $\lambda$-topology;

(4) $\left\{\Phi^{\gamma}\right\}_{\gamma \in \Gamma}$ is of type (VII);

(5) $\{s \in \mathbb{R}: 0<s<1\} \cap \operatorname{IR}_{\Gamma}(X) \neq \emptyset$ and $\{s \in \mathbb{R}: 0<s<1\} \cap \partial\left(\operatorname{IR}_{\Gamma}(X)\right) \neq \emptyset$; and

(6) for any $\gamma_{0} \in \partial\left(\operatorname{IR}_{\Gamma}(X)\right)$, we have $\gamma_{0} \in \partial\left(\operatorname{IR}_{\Gamma}(X)\right) \subset \operatorname{CR}_{\Gamma}(X)=\partial\left(\operatorname{FSR}_{\Gamma}(X)\right)$ and $\gamma \mapsto h_{\Phi \gamma}$ is not real-analytic in any neighborhood of $\gamma_{0}$ in $\Gamma$.

Proof. It is easy to see that the first three statements hold. Moreover, if $\gamma \in \Gamma$ and $|\gamma|$ is small enough, then $\gamma \in \operatorname{IR}_{\Gamma}(X)$. Since $\gamma \in \operatorname{SR}_{\Gamma}(X)$ if $\gamma$ is close enough to 1 , statement (4) follows from Theorem 5.2. By the same argument as that in the proof of Theorem 6.1, we can show that statements (5) and (6) hold.

We now take a brief look at a subset of $\mathrm{CR}(X) \cap \operatorname{HIFS}(X)$.

Definition 6.4. Let $X \subset \mathbb{C}$. Let $\operatorname{HSPT}(X)$ be the set of $\Phi \in \mathrm{CR}(X) \cap \operatorname{HIFS}(X)$ such that there exists an analytic family $\left\{\Phi^{\gamma}\right\}_{\gamma \in \Gamma}$ in $\operatorname{HIFS}(X)$ with $\Gamma=\{z \in \mathbb{C}:|z|<1\}$ satisfying all of the following:

$$
\text { - } \Phi^{0}=\Phi \text {; }
$$


- $\gamma \mapsto \Phi^{\gamma} \in \operatorname{HIFS}(X)$ is continuous with respect to the $\lambda$-topology;

- $\left\{\Phi^{\gamma}\right\}_{\gamma \in \Gamma}$ is of type (VII);

- $0 \in \partial\left(\operatorname{IR}_{\Gamma}(X)\right) \subset \mathrm{CR}_{\Gamma}(X)=\partial\left(\operatorname{FSR}_{\Gamma}(X)\right)$; and

- $\gamma \mapsto h_{\Phi^{\gamma}}$ is not real-analytic in any neighborhood of 0 .

"HSPT" stands for "Holomorphic Simultaneous Phase Transition".

Theorem 6.5. Let $X \subset \mathbb{C}$. Then $\operatorname{HSPT}(X)$ is a dense subset of $\operatorname{HIFS}(X)$ when this latter is endowed with the metric of pointwise convergence.

Proof. This proposition follows immediately from combining the argument in the proof of Lemma 4.10 in [17] (taking the similitude $S^{n}$ so that $S^{n}(X)$ is included in a small ball in $\left.\varphi_{n}(X)\right)$ with Theorem 5.2 .

Finally, we show that every type of analytic family described in Theorem 5.2 exists.

Proposition 6.6. Let $X \subset \mathbb{C}$. Then every type of analytic family in $\operatorname{HIFS}(X)$ described in Theorem 5.2 exists.

Proof. By Lemma 3.2(i) in [17], there exist families of type (I), (III), (VI) and (VIII). By Theorem 6.5, there exists a family of type (VII). Taking an appropriate subfamily of type (VII), we obtain a family of type (IV). In order to construct a family of type (II), let $\Phi=\left\{\varphi_{i}\right\}_{i \in I} \in \operatorname{SIFS}(X) \cap \operatorname{CFR}(X)$. Let $z_{0} \in \operatorname{Int}(X)$ and let $\alpha_{\gamma}(z)=\gamma\left(z-z_{0}\right)+z_{0}$ for each $\gamma \in \mathbb{C}$. Let $r>0$ be a small number such that for each $\gamma \in B(0, r)$, we have $\alpha_{\gamma}(X) \subset \operatorname{Int}(X)$. Let $\Gamma=B(0, r) \backslash\{0\}$ and let $\Psi^{\gamma}=\left\{\alpha_{\gamma} \circ \varphi_{i}\right\}_{i \in I}$ for each $\gamma \in \Gamma$. Then $\left\{\Psi^{\gamma}\right\}_{\gamma \in \Gamma}$ is an analytic family in $\operatorname{HIFS}(X)$ such that $\gamma \mapsto \Psi^{\gamma}$ is continuous with respect to the $\lambda$-topology. Moreover, $P(\gamma, t)=P_{\Phi}(t)+t \log |\gamma|$ for all $\gamma \in \Gamma$ and all $t \geq 0$. Hence, $\left\{\Psi^{\gamma}\right\}_{\gamma \in \Gamma}$ is of type (II).

We now construct a family of type (V). Let $S$ be a similarity of $\mathbb{C}$ such that $S(X) \subset \mathbb{D}$. Let $U:=\operatorname{Int}(S(X))$ and let $z_{1}, z_{2}, z_{3} \in U$ be mutually distinct points. Let $\left\{\Phi^{\gamma}\right\}_{\gamma \in \Gamma}$ be an analytic family of type (V) in $\operatorname{HIFS}(\overline{\mathbb{D}})$ such that $\varphi_{i}^{\gamma}(\overline{\mathbb{D}}) \subset U$ for all $\gamma \in \Gamma$ and all $i \in I$, as described in Example 5.4. For each $\gamma \in \Gamma$ and each $i \in I$, let $\tilde{\varphi}_{i}^{\gamma}:=S^{-1} \circ \varphi_{i}^{\gamma} \circ S$ and let $\tilde{\Phi}^{\gamma}:=\left\{\tilde{\varphi}_{i}^{\gamma}\right\}_{i \in I}$. Then $\left\{\tilde{\Phi}^{\gamma}\right\}_{\gamma \in \Gamma}$ is an analytic family in $\operatorname{HIFS}(X)$ such that $\gamma \mapsto \tilde{\Phi}^{\gamma}$ is continuous with respect to the $\lambda$-topology. Since $\left\{\Phi^{\gamma}\right\}_{\gamma \in \Gamma}$ is of type (V), Lemma 3.1 in [17] implies that $\left\{\tilde{\Phi}^{\gamma}\right\}_{\gamma \in \Gamma}$ is of type $(\mathrm{V})$ as well. This completes the proof of our proposition.

\section{REFERENCES}

[1] K. Astala, Area distortion of quasiconformal mappings, Acta Math. 173 (1994), 37-60.

[2] L. Baribeau and M. Roy, Analytic multifunctions, holomorphic motions and Hausdorff dimension in IFSs, Monatsh. Math. 147 (2006), no. 3, 199-217.

[3] C. Bandt, S. Graf, Self-similar sets 7. A characterization of self-similar fractals with positive Hausdorff measure, Proc. Amer. Math. Soc. 114 (4) (1992), 995-1001.

[4] M. F. Barnsley, Fractals Everywhere, Second Edition, Academic Press, 1993.

[5] K. Falconer, Fractal Geometry: Mathematical Foundations and Applications, Wiley, 1990.

[6] R. C. Gunning, Introduction to Holomorphic functions of several variables, Vol. I: Function Theory, Wadsworth \& Brooks/Cole Mathematics Series, Brooks/Cole Publishing Company, 1990. 
[7] P. Hanus, R.D. Mauldin and M. Urbański, Thermodynamic formalism and multifractal analysis of conformal infinite iterated function systems, Acta Math. Hungarica 96 (2002), 27-98.

[8] J. E. Hutchinson, Fractals and self-similarity, Indiana Univ. Math. J. 30 (1981), 713-747.

[9] E. Hille, Analytic function theory, II, AMS Chelsea Publishing 2002, 2nd Edition.

[10] T. Kato, Perturbation theory for linear operators, Springer (1995).

[11] J. Kotus, M. Urbański, Hausdorff dimension and Hausdorff measures of Julia sets of elliptic functions, Bull. London Math. Soc. 35 (2003), 269-275.

[12] R. D. Mauldin and M. Urbański, Dimensions and measures in infinite iterated function systems, Proc. London Math. Soc. 73 (3) (1996), no. 1, 105-154.

[13] R. D. Mauldin, M. Urbański, Conformal iterated function systems with applications to the geometry of continued fractions, Trans. Amer. Math. Soc. 351 (1999), 4995-5025.

[14] R. D. Mauldin, M. Urbański, Graph Directed Markov Systems: Geometry and Dynamics of Limit Sets, Cambridge University Press, 2003.

[15] V. Mayer, Urbański, Thermodynamical Formalism and Multifractal Analysis for Meromorphic Functions of finite order, to appear in Memoirs of AMS, also available at http://www.math.unt.edu/ urbanski/papers.html

[16] M. Roy, M. Urbański, Regularity properties of Hausdorff dimension in infinite conformal IFSs, Ergod. Th. \& Dynam. Sys. 25 (2005), no. 6, 1961-1983.

[17] M. Roy, H. Sumi, M. Urbański, Lambda-Topology vs. Pointwise Topology, to appear in Ergod. Th. \& Dynam. Sys. and available at http://www.math.unt.edu/ urbanski/papers.html

[18] M. Roy, M. Urbański, Real analyticity of Hausdorff dimension for higher dimensional graph directed Markov systems, Math. Z. 260 (1) September, 2008, pp 153-175.

[19] W. Rudin, Functional Analysis, McGraw-Hill, Inc. (1991) 2nd Edition.

[20] D. Ruelle, Repellers for real analytic maps, Ergod. Th. \& Dynam. Sys. 2 (1982), no. 1, 99-107.

[21] B. Stratmann, M. Urbański, Diophantine Extremality of the Patterson Measure, Math. Proc. Cambridge Phil. Soc. 140 (2006), 297-304.

[22] B. Stratmann, M. Urbański, Pseudo-Markov systems and infinitely generated Schottky groups, Amer. J. Math. 129 (2007), 1019-1062.

[23] M. Urbański, Porosity in conformal infinite iterated function systems, J. Numb. Theory, 88 No. 2 (2001), 283-312.

[24] M. Urbański, Diophantine Approximation of Self-Conformal Measures, Journal Number Th. 110 (2005) 219-235.

[25] M. Urbański, Diophantine Approximation for Conformal Measures of One-Dimensional Iterated Function Systems, Compositio Math. 141 (2005), 869-886.

[26] M. Urbański, Finer Diophantine and regularity properties of 1-Dimensional Parabolic IFS, Real Analysis Exch. 31 (2006), 143-165.

[27] M. Urbański, Analytic families of semihyperbolic generalized polynomial-like mappings, preprint available at http://www.math.unt.edu/ urbanski/papers.html

[28] A. Schief, Separation properties for self-similar sets, Proc. Amer. Math. Soc. 122 (1994), 111-115.

[29] M. Urbański, A. Zdunik, Real analyticity of Hausdorff dimension of finer Julia sets of exponential family, Ergod. Th. \& Dynam. Sys. 24 (2004), 279-315. 
Mario Roy, Glendon College, York University, 2275 Bayview Avenue, Toronto, Canada, M4N 3M6;

mroy@gl . yorku . ca

Webpage: www.glendon.yorku.ca/mathematics/profstaff.html

Hiroki Sumi, Department of Mathematics, Graduate School of Science, Osaka University, 1-1 Machikaneyama, Toyonaka, Osaka, 560-0043, Japan;

sumi@math.sci.osaka-u.ac.jp

Webpage: www.math.sci.osaka-u.ac.jp/ sumi/

Mariusz Urbański, Department of Mathematics, University of North Texas, Denton, TX 76203-1430, USA;

urbanski@unt.edu

Webpage: www.math.unt.edu/ urbanski 